UWThPh-2012-19

IFIC/12-62

DESY $12-155$

\title{
Renormalization Group Improved Bottom Mass from $\Upsilon$ Sum Rules at NNLL Order
}

\author{
André H. Hoang ${ }^{\natural}$, Pedro Ruiz-Femeníd ${ }^{\natural 1,2}$ and Maximilian Stahlhofen ${ }^{\complement 1,3}$ \\ ${ }^{1}$ University of Vienna, Faculty of Physics, \\ Boltzmanngasse 5, A-1090 Wien, Austria \\ ${ }^{2}$ IFIC, Universitat de València - CSIC, \\ Apt. Correus 22085, E-46071 Valencia, Spain \\ ${ }^{3}$ DESY Theory Group, Notkestraße 85, D-22607 Hamburg, Germany
}

\begin{abstract}
We determine the bottom quark mass from non-relativistic large- $n \Upsilon$ sum rules with renormalization group improvement at next-to-next-to-leading logarithmic order. We compute the theoretical moments within the vNRQCD formalism and account for the summation of powers of the Coulomb singularities as well as of logarithmic terms proportional to powers of $\alpha_{s} \ln (n)$. The renormalization group improvement leads to a substantial stabilization of the theoretical moments compared to previous fixed-order analyses, which did not account for the systematic treatment of the logarithmic $\alpha_{s} \ln (n)$ terms, and allows for reliable single moment fits. For the current world average of the strong coupling $\left(\alpha_{s}\left(M_{Z}\right)=0.1183 \pm 0.0010\right)$ we obtain $M_{b}^{1 S}=4.755 \pm 0.057_{\text {pert }} \pm 0.009_{\alpha_{s}} \pm 0.003_{\exp } \mathrm{GeV}$ for the bottom $1 \mathrm{~S}$ mass and $\bar{m}_{b}\left(\bar{m}_{b}\right)=4.235 \pm 0.055_{\text {pert }} \pm 0.003_{\text {exp }} \mathrm{GeV}$ for the bottom $\overline{\mathrm{MS}}$ mass, where we have quoted the perturbative error and the uncertainties from the strong coupling and the experimental data.
\end{abstract}

\footnotetext{
a Electronic address: Andre.Hoang@univie.ac.at

b Electronic address: Pedro.Ruiz@ific.uv.es

c Electronic address: maximilian.stahlhofen@desy.de
} 


\section{INTRODUCTION}

Determinations of the bottom quark mass $m_{b}$ have a long history and belong to the early precision analyses carried out in QCD that required higher order computations in the framework of the operator product expansion (OPE) [1, 2]. Phenomenologically they are an important input in the theoretical description of semi-inclusive B-meson decays and studies of $\Upsilon$-mesons, but they also have some relevance for Higgs decays into $b$-jets or unification and beyond the standard model studies. Apart from their phenomenological relevance, precision determinations of heavy quark masses are also a reflection of the ability to do precision computations for the quantities from which they are extracted, so that continued work in refining the respective theoretical methods and cross checks with other determination methods are warranted. Since quark masses are not physical observables due to the confinement, which binds quarks into hadronic states, they represent renormalizationdependent formal field theoretic quantities that are defined through the renormalization conditions imposed to remove the ultraviolet divergences in loop computations. Since the achievable precision in the bottom quark mass is at the percent level, and thus substantially smaller than the hadronization scale $\Lambda_{\mathrm{QCD}} \sim 200 \mathrm{MeV}$, it is mandatory to adopt so-called short-distance mass schemes for the bottom quark mass, which are free of $\mathcal{O}\left(\Lambda_{\mathrm{QCD}}\right)$ infrared renormalon ambiguities. Short-distance mass schemes involve infrared subtractions in order to reduce the sensitivity of the perturbative series to small momenta. Thus short-distance masses either have an intrinsic or an explicit dependence on the infrared subtraction scale which should be compatible with the typical short-distance scale governing the heavy quark mass-dependent observable [3-5].

One of the classic methods to determine the bottom quark mass are the $\Upsilon$ sum rules. They involve moments $P_{n}$ of the total bottom pair production cross section in $e^{+} e^{-}$annihilation through a virtual photon, defined by

$$
P_{n}=\int \frac{d s}{s^{n+1}} R_{b \bar{b}}(s)
$$

where $R_{b \bar{b}}=\sigma\left(e^{+} e^{-} \rightarrow b \bar{b}+X\right) / \sigma_{\mathrm{pt}}$ is the inclusive bottom pair production cross section and $\sigma_{\mathrm{pt}}=4 \pi \alpha^{2} / 3 s$ is the point cross section. Conceptually the moments $P_{n}$ are related to the coefficients of the zero-momentum expansion of the photon vacuum polarization function coming from $b \bar{b}$ quark pairs [6]

$$
P_{n}=\left.\frac{4 \pi^{2} Q_{b}^{2}}{n ! q^{2}}\left(\frac{d}{d q^{2}}\right)^{n} \Pi_{\mu}^{\mu}(q)\right|_{q^{2}=0},
$$

which can be determined from an OPE where the power series is dominated by the leading perturbative contribution and the power corrections represent an expansion in $\Lambda_{\mathrm{QCD}}$ over the short-distance scale $m_{b}$.

Since for large values of $n$ the energy range $\Delta E$, where $R_{b \bar{b}}$ can effectively contribute to $P_{n}$ in Eq. (1), scales as $m_{b} / n$ [7], the scale $m_{b} / n$ emerges as an additional short-distance scale in the OPE and the values of $n$ one can use for reliable perturbative analyses are limited up to around 10. Concerning the methods required for theoretical computations, two regions in $n$ can be distinguished. For $n \lesssim 3$ the theoretical moments are dominated by fluctuations at the scale $m_{b}$, such that the usual loop expansion is sufficient to determine the perturbative

contributions. A suitable short-distance mass for the low- $n \Upsilon$ sum rules is the $\overline{\mathrm{MS}}$ mass which has the bottom mass itself as the intrinsic infrared subtraction scale. On the experimental 
side, the continuum region for $R_{b \bar{b}}$ has a substantial contribution to the moments. Since there are sizable energy regions above the $\Upsilon$ bound state regime where there aren't any experimental measurements, one has to rely on extrapolations for the experimental moments $P_{n}^{\exp }$. Analyses available in the literature for the low- $n$ sum rules differ concerning the uncertainty associated to this extrapolation, see e.g. the analyses in Refs. [8] and [9]. There are variants of this method, called finite-energy sum rules where certain energy ranges are excluded entirely from the analysis [10]. For finite-energy sum rules systematic methods for computing power corrections in the OPE are currently unknown.

For $4 \lesssim n \lesssim 10$ the moments are dominated by the $\Upsilon$ bound state region and nonrelativistic heavy quark pair bound state dynamics. The large- $n$ sum rules are therefore also called non-relativistic or $\Upsilon$ sum rules. They are the focus of the present work. For the large- $n$ sum rules, in addition to $m_{b}$, also $m_{b} / \sqrt{n}$ and $m_{b} / n$ emerge as additional scales in association to the hard, soft and ultrasoft momentum scales known from heavy quarkonium physics, and one has to rely on non-relativistic effective field theory methods based on NRQCD [11] to determine the perturbative contributions. Suitable mass definitions belong to the class of the so-called threshold masses, which have the soft scale $m_{b} / \sqrt{n}$ as the intrinsic infrared subtraction scale. Several threshold masses have been defined in the literature [3] 5 , 12]. Concerning experimental data, no extrapolations are required, since the experimentally very well measured $\Upsilon$ resonances entirely dominate the moments $P_{n}^{\exp }$ and the continuum contributions of $R_{b \bar{b}}$ where no measurements exist are strongly suppressed. For a summary of various bottom mass determinations we refer to Ref. [13].

In this work we are concerned with the large- $n$ non-relativistic sum rules. Because of the additional scales, perturbation theory is a simultaneous expansion in $\alpha_{s}$ and $1 / \sqrt{n}$, where terms proportional to powers of $\left(\alpha_{s} \sqrt{n}\right)$ or $\left(\alpha_{s} \ln (n)\right)$ count as order unity and are summed to all orders. The Coulomb singular terms $\propto\left(\alpha_{s} \sqrt{n}\right)^{k}$ are generated from ladder-type diagrams with iterated potential interactions and in the so-called fixed-order expansion only these terms are being summed systematically within the NRQCD formalism [11]. Large- $n \Upsilon$ sum rules analyses were first considered in the fixed-order expansion by Voloshin in Ref. [7] at leading-order (LO) and improved up to next-to-next-to-leading order (NNLO) in Refs. [1418] with differing treatments of the logarithmic terms and the residual higher order terms and with different choices of threshold masses. Unfortunately, the moments in the fixed-order expansion do not exhibit any good convergence properties and in Refs. [14 17] various ad hoc methods were devised to extract the bottom quark mass at NNLO with a small uncertainty. We refer to the review [19] for a comparison and comments. Although the results of these fixed-order analyses were compatible, the situation was certainly unsatisfactory. We will comment on results obtained in the fixed-order expansion in Sec. V.

In the renormalization group improved (RGI) expansion also the logarithmic terms are summed systematically with the order counting scheme

$$
P_{n} \sim \sum_{k, l}\left(\alpha_{s} \sqrt{n}\right)^{k}\left(\alpha_{s} \ln (n)\right)^{l}\left[1(\mathrm{LL}) ; \alpha_{s}, 1 / \sqrt{n}(\mathrm{NLL}) ; \alpha_{s}^{2}, \alpha_{s} / \sqrt{n}, 1 / n(\mathrm{NNLL}) ; \ldots\right]
$$

for the leading logarithmic (LL), next-to leading logarithmic (NLL) and next-to-next-to leading logarithmic (NNLL) order. The RGI computations can be carried out in extensions of the original NRQCD formalism. The pNRQCD formalism [20] is based on the static expansion and factorizes soft and ultrasoft modes. The vNRQCD formalism [21] is based on the correlation of soft and ultrasoft modes through the energy-momentum dispersion relation for dynamical heavy quarks. Both formalisms are orthogonal concerning their conceptual 
basis and have different Lagrangian formulations, but results which became available within both formalisms agree.

Prior to this work a RGI bottom quark mass determination in the RS-scheme was carried out in Ref. [22] (see also [23]) at NLL order. This analysis also contained NNLL order matrix element corrections, but not the NNLL order corrections to the renormalization group evolution of the dominant quark pair production current which directly affect the normalization of the moments $P_{n}$. The NNLL order corrections to the current anomalous dimension have a contribution from genuine three-loop diagrams, called non-mixing terms, and a contribution from the subleading running of the Wilson coefficients entering the NLL anomalous dimension, called mixing terms. The ultrasoft contributions to the subleading running of the relevant coefficients were determined recently in Refs. [24, 25] in vNRQCD and in Ref. [26] in pNRQCD. Although the computations differed substantially concerning their conceptual setup and computational effort, the results from vNRQCD and pNRQCD agree. In Refs. [24, 25] also the complete set of the NNLL ultrasoft mixing contributions to the current anomalous dimension were given. The complete set of non-mixing terms has been determined already some time ago in Ref. [27 in vNRQCD, and for the consistent computation the correlated treatment and the simultaneous presence of soft and ultrasoft modes was essential. This is due to a term $\propto \ln \left(\mu_{S}^{2} / m_{b} \mu_{U}\right)$ appearing in the anomalous dimension, which enforces the relation $\mu_{U} \propto \mu_{S}^{2} / m_{b}$ between the soft renormalization scale $\mu_{S}$ and the ultrasoft renormalization scale $\mu_{U}$. The corresponding calculations have not yet been achieved in pNRQCD. The results for the mixing and non-mixing terms each exhibit very large corrections from ultrasoft gluons. As was found in Ref. [25], these large ultrasoft contributions cancel to a large extent in the sum, confirming a speculation made in Ref. [27]. Also, the known soft corrections to the current anomalous dimension, which include the complete set from the non-mixing terms [27] and the spin-dependent contributions from the mixing terms [28], are much smaller than the ultrasoft ones and even smaller than the typical residual scale dependence of the combined ultrasoft corrections [29].

In this work we determine the $1 \mathrm{~S}$ bottom quark mass [5] from the large- $n$ moments $P_{n}$ in the RGI expansion including all known NNLL order corrections, in particular the new contributions to the Wilson coefficient of the dominant quark pair production current. Our analysis is based on fits of single moments, and we examine in detail the convergence properties of the moments and their perturbative uncertainties. For the still unknown NNLL soft mixing contributions to the anomalous dimension of the dominant quark pair production current we give arguments that their size is comparable to the known soft contributions. The resulting uncertainty is tiny, and in particular smaller than the remaining scale variation of the known ultrasoft corrections. We therefore consider our analysis as NNLL order. Compared to the situation in the fixed-order expansion we find a reasonably good behavior of the perturbation series which does not require any ad hoc methods to determine the bottom quark mass.

The outline of this paper is as follows: In Sec. II we discuss the theoretical input for the non-relativistic factorization formula for the NNLL order moments we use for our analysis. For the most part we review older results, but we also discuss and numerically analyze the new NNLL order corrections to the anomalous dimension of the leading quark pair production current. An important aspect of the numerical analysis is that these NNLL order corrections are sizable. In Sec. III we therefore analyze the perturbative expansion and find that only fully expanding out all terms in the NNLL order factorization formula leads to the desired non-relativistic renormalization scaling behavior. Our numerical analysis concerning 
fits for the bottom mass determination and the details on how we implement renormalization scale variations and estimate the perturbative uncertainty are discussed in Sec. IV. In Sec. V] we discuss the results of the fits when we modify our moments to account only for the terms required in the fixed-order expansion and demonstrate the improvement achieved by the resummation of logarithms. We also present arguments that disfavor carrying out simultaneous fits with several moments using a $\chi^{2}$ function due to the strong correlation between different large- $n$ moments. Section VI contains the conclusions. We have also added three appendices giving details on the analytic results for NNLL order moments and presenting the experimental moments.

\section{THEORY MOMENTS WITH RG IMPROVEMENT}

For the determination of the theoretical expressions for the large- $n$ moments with renormalization group improvement we follow closely the approach of Refs. [7, 14, 17] to implement the simultaneous expansion in $\alpha_{s}$ and $1 / \sqrt{n}$ according to the scheme in Eq. (3), and, here, mainly concentrate on explaining the outline of the calculations and the new aspects that arise for the RG-improved calculation. The NNLL order large- $n$ moments can be determined from the integral

$$
P_{n}^{\text {th,NNLL}}=\frac{1}{4^{n}\left(M_{b}^{\text {pole }}\right)^{2 n}} \int_{E_{\mathrm{bind}}}^{\infty} \frac{d E}{M_{b}^{\text {pole }}} \exp \left\{-\frac{E}{M_{b}^{\text {pole }}} n\right\}\left(1-\frac{E}{2 M_{b}^{\text {pole }}}+\frac{E^{2}}{4\left(M_{b}^{\text {pole }}\right)^{2}} n\right) R_{\mathrm{NNLL}}^{\mathrm{thr}}(E),
$$

where $E \equiv \sqrt{s}-2 M_{b}^{\text {pole }}$ is the energy w.r.t. the pole mass threshold and $E_{\text {bind }}$ is the binding energy of the lowest lying $1 \mathrm{~S}$ resonance. Compared to the original definition of Eq. (1) the weight function $1 / s^{n+1}$ has been rewritten as an exponential expansion that explicitly separates the LL contributions from the NNLL order kinematic corrections. For the normalized $b \bar{b}$ total cross section $R$-ratio in the threshold region at NNLL order, $R_{\mathrm{NNLL}}^{\mathrm{thr}}$, one has to insert the absorptive part of the leading order and $O\left(v^{2}\right)$-suppressed non-relativistic vector current correlators arising for the heavy quark pair production process through a virtual photon in $e^{+} e^{-}$annihilation. The required results are well known from the literature on top pair production in the threshold region at a future linear collider and can be applied to the bottom pair cross section with trivial adaptations mostly related to the different number of active flavors. The explicit results can be taken from Ref. [30 32] supplemented by the modifications given in Ref. [27] arising from a more convenient convention for the $1 /(m k)$ type potentials. The contributions to the moments related to the Coulomb interaction are the same as for the fixed-order moments up to the additional logarithms summed in the leading order Coulomb potential Wilson coefficient and have been given explicitly in Ref. [14]. Deforming the energy integration into the complex plane the integral in Eq. (4) can be quite conveniently carried out by inverse Laplace transform methods. The corresponding formulae can be easily read off from tables and have been collected for convenience in Ref. [14].

The NNLL expression for the n-th moment can then be cast into the form

$$
P_{n}^{t h, \mathrm{NNLL}}=\frac{3 N_{c} Q_{b}^{2} \sqrt{\pi}}{4^{n+1}\left(M_{b}^{\text {pole }}\right)^{2 n} n^{3 / 2}}\left\{c_{1}(h, \nu)^{2} \varrho_{n, 1}(h, \nu)+2 c_{1}(h, \nu) c_{2}(h, \nu) \varrho_{n, 2}(h, \nu)\right\},
$$


where $\varrho_{n, 1}$ arises from the integration over the non-relativistic current correlator involving the dominant effective S-wave current and $\varrho_{n, 2}$ from the current correlator involving one insertion of the $\mathcal{O}\left(v^{2}\right) \sim \mathcal{O}(1 / n)$-suppressed S-wave current. Both correlators are referred to as $\mathcal{A}_{1}$ and $\mathcal{A}_{2}$, respectively, in Refs. [31, 32]. The contributions to $\varrho_{n, 1}$ coming from the Coulomb potential are UV-finite and therefore identical to the results given in Ref. [14] up to the previously mentioned trivial modification due to the Wilson coefficient of the Coulomb potential which differs from the strong coupling at NNLL order [32 34. The other contributions to $\varrho_{n, 1}$ as well as the result for $\varrho_{n, 2}$, which contribute exclusively at NNLL order, differ due to different conventions for the potentials used in Refs. [27, 32] and also because they involve UV-divergences renormalized in the $\overline{\mathrm{MS}}$ scheme (compared to a cutoff scheme used in Ref. [14]). All expressions for the non-Coulomb terms in $\varrho_{n, 1}$ and for $\varrho_{n, 2}$ have not been published before and are given explicitly in App. A. Throughout this paper all couplings and Wilson coefficients are understood to be renormalized in the $\overline{\mathrm{MS}}$ scheme.

The terms $c_{1}$ and $c_{2}$ are the Wilson coefficients of the dominant and the $\mathcal{O}\left(v^{2}\right)$ subleading currents, respectively. The NNLL order anomalous dimension of $c_{1}$ contains the previously mentioned mixing and non-mixing corrections according to Refs. [24 26] and Ref. [27, respectively. These two types of NNLL order corrections to $c_{1}$ have not yet been analyzed together in any phenomenological analysis of bottom pair production close to the threshold. A brief numerical analysis of $c_{1}$ focusing on the NNLL order scale variation and the uncertainty due to the yet unknown soft mixing contributions in the anomalous dimension of $c_{1}$ is carried out at the end of this section. Details on the analytic results for $c_{1}$ and $c_{2}$ are given at the end of this section and in App. B.

We note that in our analysis we treat the charm quark as massless. Up to now the effects of the non-zero charm mass have only been treated in the fixed-order formalism exhibiting a shift in the bottom mass between -20 and $-30 \mathrm{MeV}$ [18, 35]. The corrections from the non-zero charm mass $m_{c}$ are conceptually interesting since $m_{c}$ is numerically close to the soft scale $\sim \alpha_{s} m_{b}$ and generically above the ultrasoft scale $\sim \alpha_{s}^{2} m_{b}$. The effects are therefore not suppressed by additional factors of $m_{c}^{2} / m_{b}^{2}$ and are even somewhat enhanced since the charm mass interferes as a physical infrared scale in the renormalon cancellation. At this time a systematic renormalization group improved treatment of massive virtual quark thresholds in heavy quarkonium production is still lacking. However, taking the known effects in the fixed-order approach as a guideline, we conclude that the associated uncertainty is smaller than the perturbative uncertainties of our present analysis in Sec. IV.

The correlators and Wilson coefficients all depend on the dimensionless vNRQCD velocity renormalization evolution parameter $\nu$ that is used to implement the correlated evolution of soft $\left(\mu_{S}\right)$ and ultrasoft scales $\left(\mu_{U}\right)$ according to the non-relativistic scaling $\mu_{U} \propto \mu_{S}^{2} / m_{b}$. For the large- $n$ moments the typical choice for $\nu$ is of order of the velocity of the bottom quarks which generically scales as $1 / \sqrt{n}$. The residual dependence of predictions on changes of $\nu$ are used to estimate the perturbative uncertainty of the low-energy contributions contained in the theoretical prediction. On the other hand, changes coming from the variation of the matching scale $\mu_{h}$ can be used as an estimate of the perturbative uncertainty of the high-energy contributions contained in the moments. Both types of uncertainties can be considered as independent, because hard and low-energy contributions are separated in the non-relativistic effective theory. In order to avoid that the ultrasoft scale can exceed the soft scale we use the assignment $\mu_{S}=\nu \mu_{h}, \mu_{U}=\nu^{2} \mu_{h}$ and impose the restriction $\nu \leq 1$. Moreover we parametrize the matching scale as $\mu_{h}=h m_{b}$, where the typical choice for $h$ is of order one. The renormalization scaling parameter $h$ has also been indicated in Eq. (5). 
In our parametrization with the dimensionless renormalization scaling parameters $\nu$ and $h$ all explicit dependence on the bottom quark mass $m_{b}$ cancels from the Wilson coefficients as well as from the matrix elements corrections contained in $\varrho_{n, 1}$ and $\varrho_{n, 2}$. The only explicit dependence on the bottom quark mass arises from the overall dimensional factor shown in Eq. (5). In addition, there remains an implicit dependence on the bottom mass through the dependence of the Wilson coefficients or the matrix element corrections on the strong coupling $\alpha_{s}$, which has to be evaluated either at the matching, the soft or the ultrasoft renormalization scales. Because the matching scaling parameter $h$ always occurs together with an additional $m_{b}$ factor and because the solutions of the anomalous dimensions can be expressed in terms of the strong coupling $\alpha_{s}$ evaluated at the hard, soft or ultrasoft scales, it is straightforward to implement the $h$-dependence of the moments by simply inserting a factor of $h$ to the argument of each $\alpha_{s}$ that arises. This method covers all $h$-dependence except for two terms, one arising from the term $\ln \left(\mu_{S}^{2} / m_{b} \mu_{U}\right)=\ln h$ in the NNLL non-mixing anomalous dimension of $c_{1}\left[27\right.$ and one in the NNLL order matching condition $c_{1}\left(\mu_{h}, 1\right)$. Both terms are given in App. B as well.

The moments shown in Eq. (5) have been written in terms of the bottom quark pole mass $M_{b}^{\text {pole }}$, which is known to be sensitive to renormalon long-distance effects that seriously deteriorate the convergence of the perturbative expansion. It is therefore mandatory to employ a better defined short-distance bottom quark mass scheme. Short-distance mass schemes have an intrinsic dependence on an infrared cutoff scale [36], and for problems involving non-relativistic heavy quarkonium dynamics one has to employ so-called threshold masses, where this scale is of order of the inverse Bohr radius, $\mathcal{O}\left(m_{b} \alpha_{s}\right)$ [12. In this work we will use the $1 \mathrm{~S}$ mass defined as half the perturbative contribution to the ${ }^{3} S_{1}$ bottonium ground state, $\Upsilon(1 S)$ [37, 38]. Other possible threshold mass definitions have been discussed e.g. in Ref. [12], and in Ref. [22] the so-called renormalon-subtracted mass scheme was employed. The result can finally be converted to the $\overline{\mathrm{MS}}$ mass, which is frequently used for predicting physical quantities involving scales larger than the bottom mass. Although this conversion avoids the renormalon problem of the pole mass, we note, however, that the conversion introduces a rather large dependence on the uncertainty in the value of the strong coupling $\alpha_{s}$ due to a term $\propto \alpha_{s} m_{b}$ appearing in the conversion series. This motivates quoting the result for the $1 \mathrm{~S}$ mass as the main result of our analysis.1] We note, however, that in our case this $\alpha_{s}$-dependence just compensates for the $\alpha_{s}$-dependence of the $1 \mathrm{~S}$ mass we determine from our sum rule analysis.

To convert Eq. (5) to the 1S bottom mass scheme its relation to the pole mass is required, which at NNLL order reads

$$
M_{b}^{\text {pole }}=M_{b}^{1 S}\left\{1+\Delta^{\mathrm{LL}}+\Delta^{\mathrm{NLL}}+\left[\left(\Delta^{\mathrm{LL}}\right)^{2}+\Delta_{c}^{\mathrm{NNLL}}+\Delta_{m}^{\mathrm{NNLL}}\right]\right\} .
$$

The various $\Delta$ terms are given in Ref. [31], where it is necessary to use Eq. (A3] for the coupling parameter $a$ and the result for $\mathcal{V}_{k, \text { eff }}^{(s)}$ from Ref. [32] for the coefficient $\mathcal{V}_{k}^{(s)}$. The logarithms in the $\Delta$ terms are to be understood as $L \equiv \ln \left[\mu_{S} /\left(a m_{b}\right)\right]$, which reduces to $\ln [\nu / a]$ for $h=1$ as given in Ref. [31]. On the RHS of Eq. (6) the terms $\Delta^{\mathrm{LL}}$ and $\Delta^{\mathrm{NLL}}$ are the LL and NLL order contributions in the non-relativistic expansion, and the three terms in the brackets are the NNLL order corrections. As discussed in detail in Ref. [17], it is crucial to reexpand the series in a way that is consistent with the exponential non-relativistic

\footnotetext{
${ }^{1}$ We refer the reader to Ref. 39] for a review on the in our view incoherent situation concerning the value and uncertainty in $\alpha_{s}\left(M_{z}\right)$.
} 
expansion given in Eq. (4) where the LL order contribution to the binding energy is treated as a leading order term. This is achieved by using the expression

$$
\begin{aligned}
\frac{1}{\left(M_{b}^{\text {pole }}\right)^{2 n}}= & \frac{1}{\left(M_{b}^{1 S}\right)^{2 n}} \exp \left(-2 n \Delta^{\mathrm{LL}}\right) \\
& \times\left\{1-2 n \Delta^{\mathrm{NLL}}+n\left[\left(\Delta^{\mathrm{LL}}\right)^{2}-2 \Delta^{\mathrm{NNLL}}+2 n\left(\Delta^{\mathrm{NLL}}\right)^{2}\right]+\ldots\right\},
\end{aligned}
$$

where the first, second and third term in the second line correspond to LL, NLL and NNLL order, respectively. In order to consistently remove the infrared renormalon problem associated with the pole mass when switching to the $1 \mathrm{~S}$ scheme, it is important to consistently expand out the series in the curly brackets with the corresponding LL, NLL and NNLL order terms contained in $\rho_{n, 1}$, while the exponential term containing $\Delta^{\mathrm{LL}}$ remains unexpanded. For the $\rho_{n, 2}$ only the LL exponential term in Eq. (7) has to be employed. Note that it is also crucial to use the same values for the renormalization scale parameters $\nu$ and $h$ in the Wilson coefficients and couplings of Eqs. (5) and (7). Unless stated explicitly we always refer to the $1 \mathrm{~S}$ mass definition as the bottom quark mass: $m_{b} \equiv M_{b}^{1 S}$.

The contributions to the renormalization group evolution of $c_{1}$, the Wilson coefficient of the leading bottom pair production current, can be parametrized as

$$
\begin{aligned}
\frac{c_{1}(h, \nu)}{c_{1}(1,1)} & =\exp \left[\xi^{\mathrm{NLL}}(h, \nu)+\left(\xi_{\mathrm{m}}^{\mathrm{NNLL}}(h, \nu)+\xi_{\mathrm{nm}}^{\mathrm{NNLL}}(h, \nu)\right)+\ldots\right] \\
& =1+\xi^{\mathrm{NLL}}+\left[\left(\frac{1}{2} \xi^{\mathrm{NLL}}\right)^{2}+\xi_{\mathrm{m}}^{\mathrm{NNLL}}+\xi_{\mathrm{nm}}^{\mathrm{NNLL}}\right]+\ldots,
\end{aligned}
$$

where $\xi^{\mathrm{NLL}}$ refers to the NLL order contribution and the $\xi_{\mathrm{m}}^{\mathrm{NNLL}}$ and $\xi_{\mathrm{nm}}^{\mathrm{NNLL}}$ to the NNLL order mixing and non-mixing corrections, respectively. The matching condition $c_{1}(h, 1)$ and the expressions for the $\xi$ 's can be found in App. B and the references cited there. In the second line of Eq. (8) we have displayed the consistently expanded form where the terms in the squared brackets represent the NNLL corrections and the explicit dependence of the $\xi$ 's on the scale parameters has been suppressed. We note that since the LL order anomalous dimension for the current is zero, the expanded form does not contain any exponentiated LL order contribution.

Based on the recently completed calculation of the ultrasoft NLL running of the Wilson coefficients associated to the $\mathcal{O}\left(v^{2}\right)$ - and $\mathcal{O}\left(\alpha_{s} v\right)$-suppressed potentials [24-26], the ultrasoft mixing contributions to $\xi_{\mathrm{m}}^{\mathrm{NNLL}}$, referred to as $\xi_{\mathrm{m} \text {,usoft }}^{\mathrm{NNL}}$ has been determined in Ref. [25]. Concerning the corresponding soft mixing contributions currently only those coming from the NLL order anomalous dimension of the spin-dependent $\mathcal{O}\left(v^{2}\right)$-suppressed potential are fully known and were found to be tiny [28. On the other hand, because the NLL matching conditions of all the suppressed potentials are known, it is possible to compute the term $\propto \alpha_{s}^{3} \ln \nu$ of the soft mixing contributions, which we call $\xi_{\mathrm{m}, \mathrm{soft} 1}^{\mathrm{NNL}}$. The result was already presented in Ref. [27] and it also given in Eq. (B7).

The NNLL order non-mixing contributions in $\xi_{\mathrm{nm}}^{\mathrm{NNLL}}$, i.e. the ultrasoft and soft contributions, are both completely known already since some time from Ref. [27]. In the same publication it was observed that the ultrasoft non-mixing contributions were more than an order of magnitude larger than the soft ones, and that the smallness of the latter was not arising from any accidental cancellation between different color factors but was a genuine property of all soft non-mixing contributions. In the following we analyze all known NNLL 
contributions together numerically and give arguments suggesting that the ultrasoft contributions also dominate the mixing corrections and that the unknown soft corrections are negligible.
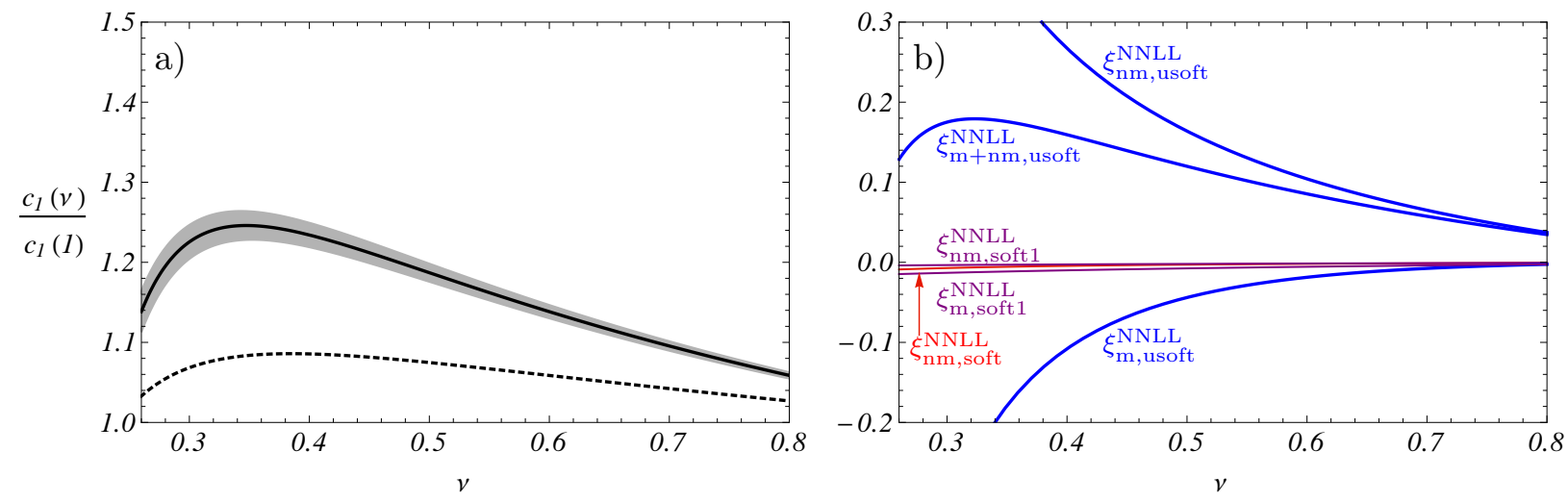

FIG. 1. Panel a): RG evolution of the ${ }^{3} S_{1}$ current coefficient $c_{1}(\nu)$ normalized to $c_{1}(1)$ for $m_{b}=$ $4.7 \mathrm{GeV}$ and $h=1$. The dashed line represents the full NLL result in expanded form: $1+\xi^{\mathrm{NLL}}$. The solid line accounts for all known contributions including the new ultrasoft NNLL mixing correction in Eq. (B2) as well as the leading soft mixing logarithm at NNLL $\xi_{\mathrm{m}, \mathrm{soft}}^{\mathrm{NNL}}$ in Eq. (B7): $1+\xi^{\mathrm{NLL}}+\left(\xi^{\mathrm{NLL}}\right)^{2} / 2+\xi_{\mathrm{nm}}^{\mathrm{NNLL}}+\xi_{\mathrm{m}, \mathrm{usoft}}^{\mathrm{NNL}}+\xi_{\mathrm{m}, \mathrm{soft} 1}^{\mathrm{NNL}}$. The gray area around the black line is generated by varying the known soft NNLL contributions to that curve by a factor between 0 and 2 . Panel $b$ ): Separate curves for the different (soft/ultrasoft, mixing/nonmixing) NNLL corrections $\left(\xi^{\mathrm{NNLL}}\right)$ to the running of $c_{1}$ as indicated in the plot. The NNLL contribution coming from the exponentiation of the NLL term, $\left(\xi^{\mathrm{NLL}}\right)^{2} / 2$, is tiny. Since it virtually coincides with the horizontal zero-line in this plot, it has not been drawn. The curve for $\xi_{\mathrm{nm}, \mathrm{soft} 1}^{\mathrm{NNL}}$ represents the linear logarithmic term $\propto \alpha_{s}^{3} \ln \nu$ contained in the complete soft non-mixing correction $\xi_{\mathrm{nm} \text {,soft }}^{\mathrm{NNLL}}$. For both plots we have used $\alpha_{s}^{\left(n_{f}=4\right)}(4.7 \mathrm{GeV})=0.217$.

In the left panel of Fig. 1 the renormalization scale evolution of $c_{1}(h, \nu) / c_{1}(1,1)$ is displayed for $0.26 \leq \nu \leq 0.8$ at NLL order (dashed line) and including all known NNLL order contributions, where the soft mixing corrections are approximated by $\xi_{\mathrm{m}, \mathrm{Noft}}^{\mathrm{NLL}}$ (solid line). The gray band drawn around the NNLL result arises from the variation of the result coming from multiplying the soft terms at NNLL order by a factor between zero and two. Comparing the width of the gray band with the overall shift between the NLL and NNLL order results and also with the overall remaining $\nu$ dependence of $c_{1}(h, \nu) / c_{1}(1,1)$ at NNLL order we make two important observations.

First, the ultrasoft contributions dominate the NNLL order result and are about an order of magnitude larger than the known soft contributions. From the right panel of Fig. 1 we see that this is true for the mixing and non-mixing contributions separately as well as for the sum. From the close vicinity of the curves for the full expression for $\xi_{\mathrm{nm}, \mathrm{soft}}^{\mathrm{NNL}}$ and its linear logarithmic contribution $\xi_{\mathrm{nm}, \mathrm{soft} 1}^{\mathrm{NNLL}} \propto \alpha_{h}^{3} \ln \nu$, given in Eq. (B9), we can also conclude that the curve for $\xi_{\mathrm{m}, \mathrm{soft} 1}^{\mathrm{NNL}}$ should represent the correct order of magnitude for the full set of soft mixing corrections. It is therefore reasonable to take the gray band as an uncertainty associated to the currently unknown soft mixing corrections. Comparing the gray band to the size and scale dependence of the bigger ultrasoft corrections we can conclude that this 
uncertainty is smaller than the remaining NNLL renormalization scale variation we observe in the relevant range $0.3 \lesssim \nu \lesssim 0.6$. This suggests that the unknown soft corrections can be neglected for a NNLL order prediction.

As the second observation, we see that the NNLL order corrections to the anomalous dimension of $c_{1}$ are about a factor two larger than the NLL order ones. This somewhat unsettling fact might cast slight doubts concerning the quality of the perturbative expansion due to large ultrasoft corrections, but might be as well associated to an anomalously small NLL order correction, which amounts to less than 10\%. Nevertheless, given the situation it is certainly appropriate to invest a closer look on the behavior of the perturbation series for the moments.

Finally we comment on the presence of nonperturbative power corrections in the OPE of the current correlators $\mathcal{A}_{i}$. The effect of the leading power correction on the theoretical large- $n$ moments in Eq. (5) is associated with the $\mathcal{O}\left(\Lambda_{\mathrm{QCD}}^{4}\right)$ gluon condensate arising in the OPE for $\mathcal{A}_{1}$. The corresponding nonperturbative power correction to the $n$-th moment can be written approximately as [7]

$$
\delta P_{n}^{t h, n p}=P_{n}^{t h, \mathrm{LL}}\left\langle\alpha_{s} \mathbf{G}^{2}\right\rangle \frac{n^{3} \pi}{72 m_{b}^{4}} \exp \left(-0.4 C_{F} \alpha_{s} \sqrt{n}\right),
$$

where we take $\alpha_{s}$ in the exponent at the soft scale. To our knowledge the subleading corrections to the Wilson coefficient of the gluon condensate are currently unknown. Numerically the relative correction from Eq. (9) is less than a percent for $n<20$, i.e. even smaller then the experimental uncertainty and therefore negligible for our purpose, cf. Sec. IV. In order to make sure that also higher order power corrections [40] are sufficiently suppressed, we, however, only consider $n \lesssim 10$ for our analysis.

\section{THE PERTURBATIVE EXPANSION}

The perturbative series for the large- $n$ moments $P_{n}^{\text {th }}$ in Eq. (5) follows the non-relativistic expansion scheme of Eq. (3) and, as explained in the previous section, requires that the perturbative series for $\rho_{n, 1}$ coming from the leading order current correlator is consistently expanded with the additional corrections of Eq. (7) that arise from employing the 1S threshold mass scheme. However, concerning the factor $c_{1}^{2}$ in Eq. (5) one has the option either to keep it as an overall factor for the series associated to $\varrho_{n, 1}$ ("factorized form") or to expand it out as well together with the series of $\varrho_{n, 1}$ ("expanded form"). The difference between the two ways to expand $P_{n}^{t h}$ represents terms from beyond NNLL order and should, in principle, be within the theoretical uncertainty of our NNLL result. However, given the observations concerning the perturbative behavior of $c_{1}$ discussed above we believe that it is mandatory to have a closer look on the expanded and factorized expansions of the moments.

An important property of the large- $n$ moments is that the non-relativistic dynamics encoded in them is governed by bottom quark velocities $v \sim \mathcal{O}(1 / \sqrt{n})$. Since the vNRQCD renormalization scaling parameter $\nu$ is typically of order $v$, we would expect that a good convergence of the series and the best physical description is achieved for $\nu \sim(1 / \sqrt{n}+$ const.), where the additional constant term arises from a saturation effect that protects the renormalization scale from vanishing for large $n t^{2}$ Next we examine for the expanded and the factorized form of the large- $n$ moments, how well they satisfy this behavior.

${ }^{2}$ As argued in Ref. [7] the proper choice for the soft $\left(\mu_{S}\right)$ and ultrasoft renormalization scale $\left(\mu_{U}\right)$ in the limit $n \rightarrow \infty$ approaches the inverse Bohr radius $\left(\sim \alpha_{s} m_{b}\right)$ and the binding energy $\left(\sim \alpha_{s}^{2} m_{b}\right)$ of the lowest lying resonance, respectively. 
In Fig. 2 we plot the values of the renormalization parameter $\nu$ that are required to satisfy $P_{n}^{t h}\left(m_{b} ; \nu\right)=P_{n}^{\exp }$ as a function of $n$ using $h=1$ and a fixed value of $m_{b}$, where the $P_{n}^{\exp }$ are the experimental moments. The value of the bottom quark mass has been fixed by fitting the theoretical 10-th moment, $P_{10}^{t h}$, to the experimental one using $\nu=f(1 / \sqrt{10}+0.2)$. Here the constant $f$ is a fudge parameter which should be of order one and which we have varied between 0.8 and 1.25 in steps of 0.05 . The results for $\nu$ we obtained for $n \neq 10$ are shown as blue dots, and values for equal $f$ are connected by the blue lines. The left panel shows the outcome for the expanded moments and the right panel for the factorized moments. We see that the $\nu$ values for the fully expanded moments decrease with $n$ and are indeed consistent with the expected behavior $\nu \sim(1 / \sqrt{n}+$ const. $)$. On the other hand, the $\nu$ values from the factorized moments increase with $n$ and are inconsistent with the expected behavior. As a comparison we have displayed the functions $\nu=f(1 / \sqrt{n}+0.2)$ with $f=0.8,1.0,1.25$ as the dashed red lines.
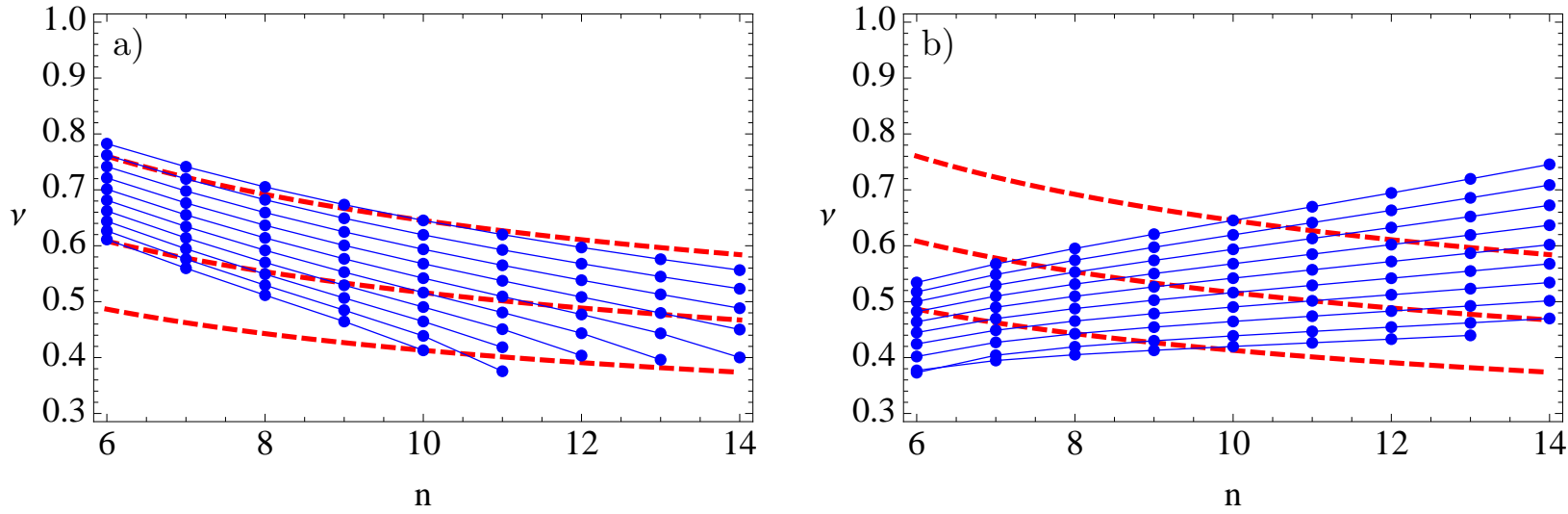

FIG. 2. Values for the renormalization parameter $\nu$ required by $P_{n}^{t h}\left(m_{b} ; \nu\right)=P_{n}^{\text {exp }}$ for fixed mass $m_{b}$ and different $n$. The blue lines connect the $\nu$ values (blue dots) for the same reference mass. Lines for 10 different reference masses determined from $P_{10}^{t h}\left(m_{b} ; \nu=f(1 / \sqrt{10}+0.2)\right)=P_{10}^{\text {exp }}$ with $f$ between 0.8 and 1.25 are shown. For the plot in panel $a$ ) we used the fully expanded NNLL expression and for the plot in panel $b$ ) the factorized NNLL expression for $P_{n}^{t h}$, as described in the text. The reference masses in a) range from $4.77 \mathrm{GeV}$ for $f=0.8$ to $4.74 \mathrm{GeV}$ for $f=1.25$. The reference masses in b) range from $4.69 \mathrm{GeV}$ for $f=0.8$ to $4.64 \mathrm{GeV}$ for $f=1.25$. The dashed red curves are defined by $\nu=f(1 / \sqrt{n}+0.2)$ with $f=0.8,1,1.25$ respectively.

The quite different outcome for the expanded and factorized moments is caused by the perturbative behaviour of $c_{1}$ discussed in the previous section. This shows that the (expansion scheme dependent) fraction of terms from beyond the NNLL level included in the two ways of the NNLL expansion for the moments, are large enough to qualitatively affect renormalization scaling features of the perturbative result. Because only the fully expanded moments show the expected behavior for their renormalization scaling parameter $\nu$, we adopt them for our numerical analysis to determine the bottom quark mass. However, we require as an important cross check that the bottom mass values determined from the factorized moments have to be consistent with those from the expanded moments within the perturbative uncertainty. 


\section{NUMERICAL ANALYSIS}

The experimental moments we use for our analysis are based on the measurements of the masses and electromagnetic decay widths of the first four $\Upsilon$ resonances $(\Upsilon(1 S)-\Upsilon(4 S))$ [13] in the narrow width approximation, BABAR data for the threshold region [41] in the energy range between $\sqrt{s}=10.62$ and $\sqrt{s}=11.21$ and the perturbative QCD result for the continuum at energies $\sqrt{s}>11.21$ [42], where no experimental data is available ${ }^{3}$ As we are aiming at an analysis for large- $n$ moments, the $\Upsilon$ resonances and the BABAR region constitute the major part of the experimental moments $((87,93,96,98) \%$ for for the $\Upsilon$ resonances, and $(5.7,3.7,2.3,1.4) \%$ for the BABAR region for $n=(6,8,10,12))$ and only little effort has to be invested for the continuum region above $11.21 \mathrm{GeV}$ where no experimental data exists. We follow the approach of Ref. [43] for the BABAR region and also adopt an additional $10 \%$ model uncertainty for the contiuum region. We stress, however, that the latter uncertainty only constitutes a very small and numerically irrelevant part of the whole error budget of the large- $n$ moments which are dominated by the uncertainties from the $\Upsilon$ resonances and the BABAR region. We note that applying our approach for low- $n$ moments, we obtain results that are perfectly compatible with the moments given in Ref. [44] albeit with somewhat larger errors if applied for low $n$ values, as in their approach only perturbative QCD uncertainties were accounted for in the continuum region. The mean values together with the respective statistical and systematic errors of the $P_{n}^{\exp }$ for $4 \leq n \leq 14$ are given in App. C,

In order to determine the $1 \mathrm{~S}$ bottom mass from single moment fits we simply solve the equation $P_{n}^{t h}\left(m_{b}\right)=P_{n}^{e x p}$ for $m_{b}$. In order to estimate the perturbative error we carry out these fits many times varying matching and renormalization scales in reasonable ranges. To parametrize the scale variations for the different moments $P_{n}^{\text {th }}$ such that the $n$-dependence of the renormalization scales and their correlation is implemented coherently we use the assignments

$$
\mu_{h}=h m_{b}, \mu_{S}=h m_{b} f \nu^{*} \quad \text { and } \quad \mu_{U}=h m_{b}\left(f \nu^{*}\right)^{2}
$$

for the matching, soft and ultrasoft scales, respectively, where $\nu^{*}=(1 / \sqrt{n}+0.2)$ is our default choice for the renormalization scaling parameter $\nu$. So the matching, soft and ultrasoft scales adopt their default values for $h=f=1$, and we parametrize variations of these scales by scanning over a certain region in the two-dimensional $h$ - $f$-plane.

The contour plot in Fig. 3 shows the results for the $1 \mathrm{~S}$ bottom mass obtained for $n=10$ at NNLL order using the expanded expression for the theoretical moment in the $h$ - $f$-plane. The default values are $h=f=1$ (red dot). For estimating the perturbative error we vary $h$ and $f$ around their default values applying the restriction that the ultrasoft renormalization scale remains within the range $0.5 m_{b} \nu^{* 2} \leq \mu_{U} \leq 2 m_{b} \nu^{* 2}$. Furthermore we fix the range for the variation of $h$ to $0.75 \leq h \leq 1 / 0.75$. The two conditions define the region indicated by the red dashed lines, and we note that for both $h$ and $f$ being small this region restricts the ultrasoft scale from dropping below $0.13 m_{b}$ for $n=10$.

Figure 3 displays the overall behavior for the bottom mass extracted from the NNLL order moments, indicating steeply rising mass values for decreasing values of $h$ and $f$ corresponding to the matching, soft and ultrasoft scales being small. However, in the restricted region we

${ }^{3}$ We thank V. Mateu and B. Dehnadi for providing us the data compilation and a numerical code for the experimental moments. 


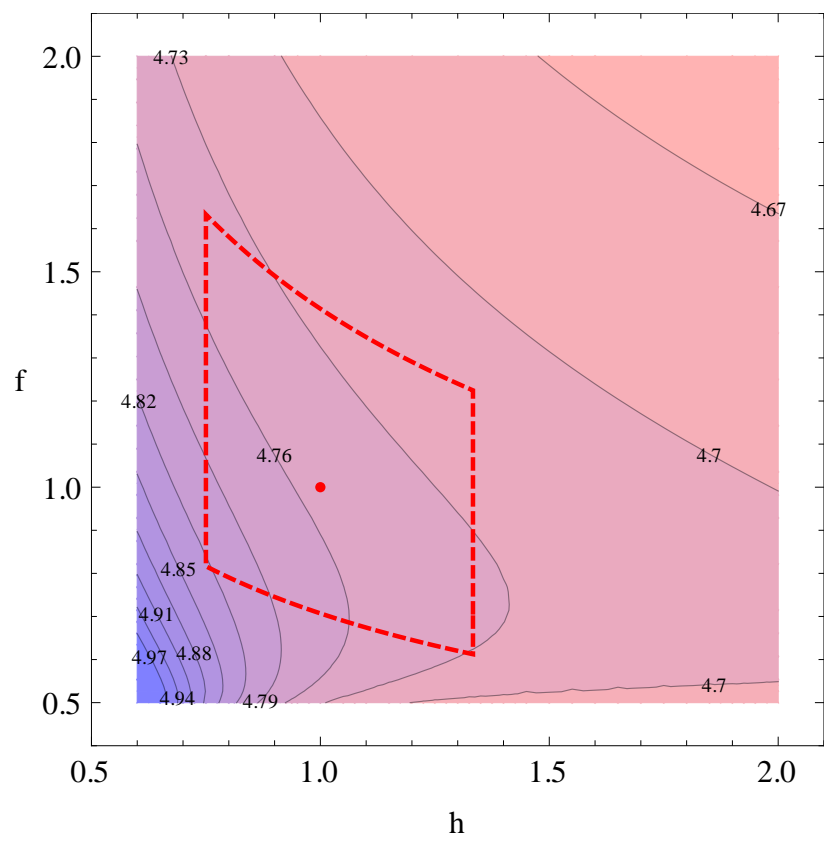

FIG. 3. Contour plot of the $1 \mathrm{~S}$ bottom mass determined from $P_{10}^{t h}\left(m_{b}\right)=P_{10}^{e x p}$ as a function of the parameters $h$ and $f$ as defined in the text. The different contours are labeled by the respective mass value in $\mathrm{GeV}$. The region in the $h-f$ plane bounded by the red dashed line represents the parameter space we scan to determine the variation of the mass, which contributes to our perturbative error estimate. The region is defined by $0.75 \leq h \leq 1 / 0.75$ and demanding that $0.5 \mu_{U}^{*} \leq \mu_{U} \leq 2 \mu_{U}^{*}$, where $\mu_{U}^{*}=m_{b} \nu^{* 2}$. The red point inside this area indicates our default values $f=h=1$ for the mass determination.

use for estimating the perturbative error, the contours are relatively flat with the default $m_{b}$ representing a central value within the overall range of values obtained in the restricted region.

For comparison we have also displayed in Fig. 4 the corresponding bottom mass contour plots obtained from the LL order moment (left panel) and from the NLL order moment (right panel). It is a conspicuous fact that in the restricted $h$ - $f$ region the form of the contours and the range of the bottom masses covered for the LL analysis is quite similar to the NNLL order analysis. On the other hand, for the NLL order analysis the outcome is quite different, since the mass values strongly decrease in the small $h$ - $f$ region and since the default bottom mass is very close to the upper end of the obtained range of bottom mass values. Moreover there is hardly any overlap to the mass range obtained from the NNLL and LL analyses. The overall behavior we see from the outcome of this analysis at LL, NLL and NNLL order confirms the slightly marginal character of the perturbative series for the large- $n$ moments we already discussed in Sec. III.

From our observations it is clear that one has to conclude that the LL and NLL order renormalization scale variations do not provide reliable estimations of the perturbative uncertainty at their respective orders and that the uncertainties are in fact substantially larger. On the other hand, the consistency of the analysis at LL and NNLL order and between the NNLL order bottom mass results for the expanded and factorized moments, as shown below, are indications that the NNLL analysis is reliable and that the variations 

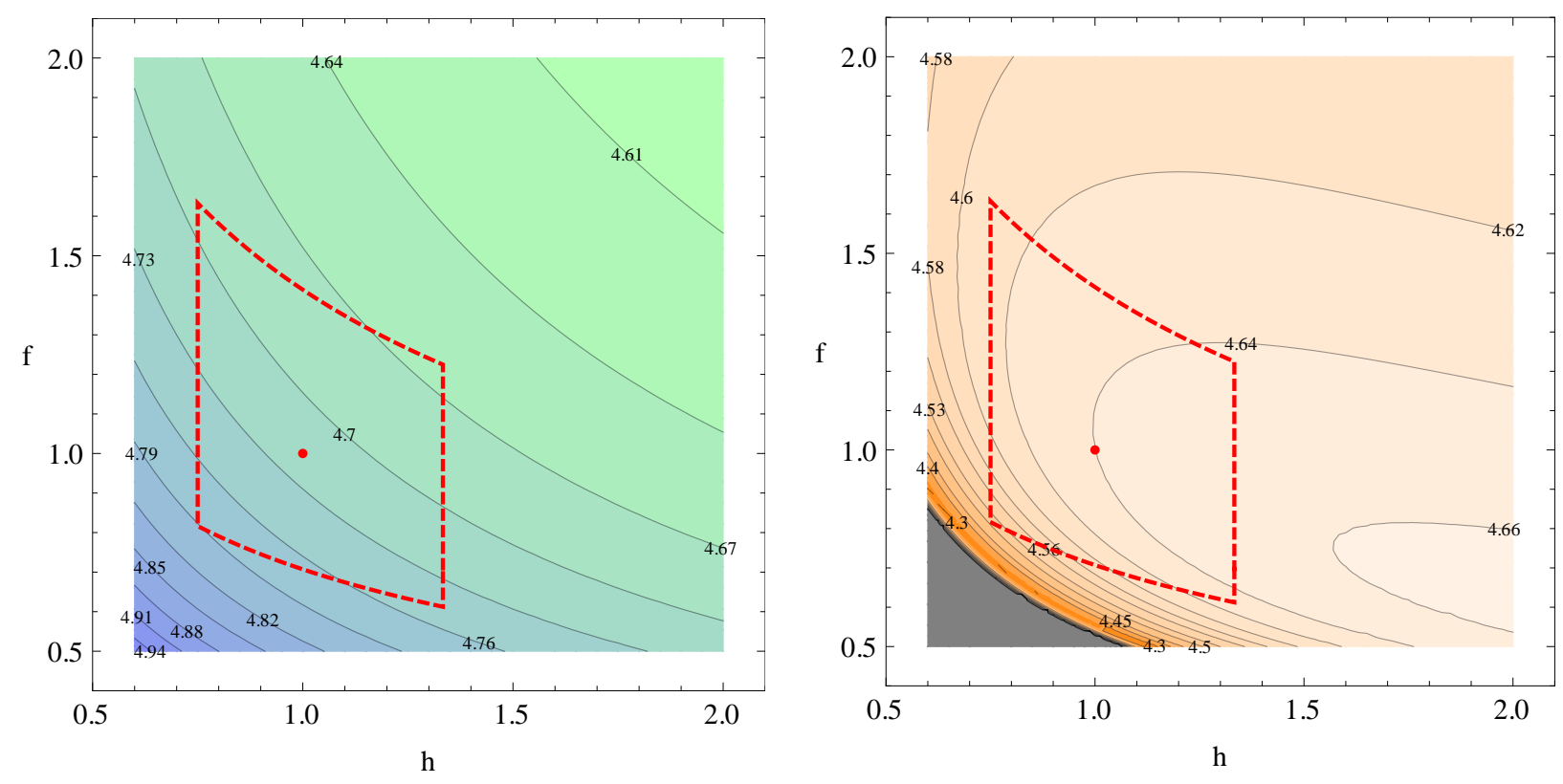

FIG. 4. Contour plots of the $1 \mathrm{~S}$ bottom mass contour plots determined from the 10th moment at LL (left panel) and NLL (right panel) order. The gray region in the lower left corner of the NLL plot indicates that the equation $P_{10}^{t h, N L L}\left(m_{b}\right)=P_{10}^{\exp }$ does not have a solution related to the ultrasoft scale being too small $\left(\mu_{U} \lesssim 0.6 \mathrm{GeV}\right)$.

of $f$ and $h$ in the region described above give a proper estimate of the perturbative NNLL order uncertainty. We therefore adopt the NNLL order large- $n$ moments and the procedure as described above to determine the bottom mass and the perturbative uncertainty for our final numerical analysis following below.

In Fig. 5 we present the results for the $1 \mathrm{~S}$ bottom mass from the moments for $n=$ $6,8,10,12$ in the expanded form as a function of $\alpha_{s}\left(M_{Z}\right)$ at NNLL order (red) and for comparison also at LL (blue) and NLL order (orange). Given the discussion above we will, however, only discuss the NNLL order results. The respective dashed lines represent the mass for the default scale choices with $f=h=1$ and the bands come from varying $f$ and $h$ in the region as discussed in detail above. The masses obtained from the default scale choices are remarkably consistent for the different $n$ values exhibiting deviations of less than $20 \mathrm{MeV}$ for $\alpha_{s}\left(M_{Z}\right)$ in the region around 0.118 , where the current world average $\alpha_{s}\left(M_{Z}\right)=0.1183 \pm 0.0010$ [39], is located. The width of the band from the scale variations, on the other hand, slightly decreases for increasing $n$ indicating that the dependence of the moment normalization on the exponent of the mass slightly overcompensates the increase of higher order $\alpha_{s}$ corrections due to the smaller renormalization scales. Overall, the 1S mass slightly increases with $\alpha_{s}$, but the dependence is rather weak and linear to a very good approximation so that the error in $\alpha_{s}\left(M_{Z}\right)$ only has a rather small impact on the final uncertainty in the bottom mass. We note that the uncertainties in the bottom mass coming from the error of the experimental moments amount to $(6.5,4.3,3.3,2.7) \mathrm{MeV}$ for $n=(6,8,10,12)$ and are much smaller than the perturbative error and the uncertainty coming from $\alpha_{s}$. For quoting the final result the experimental uncertainty does not play any essential role. 

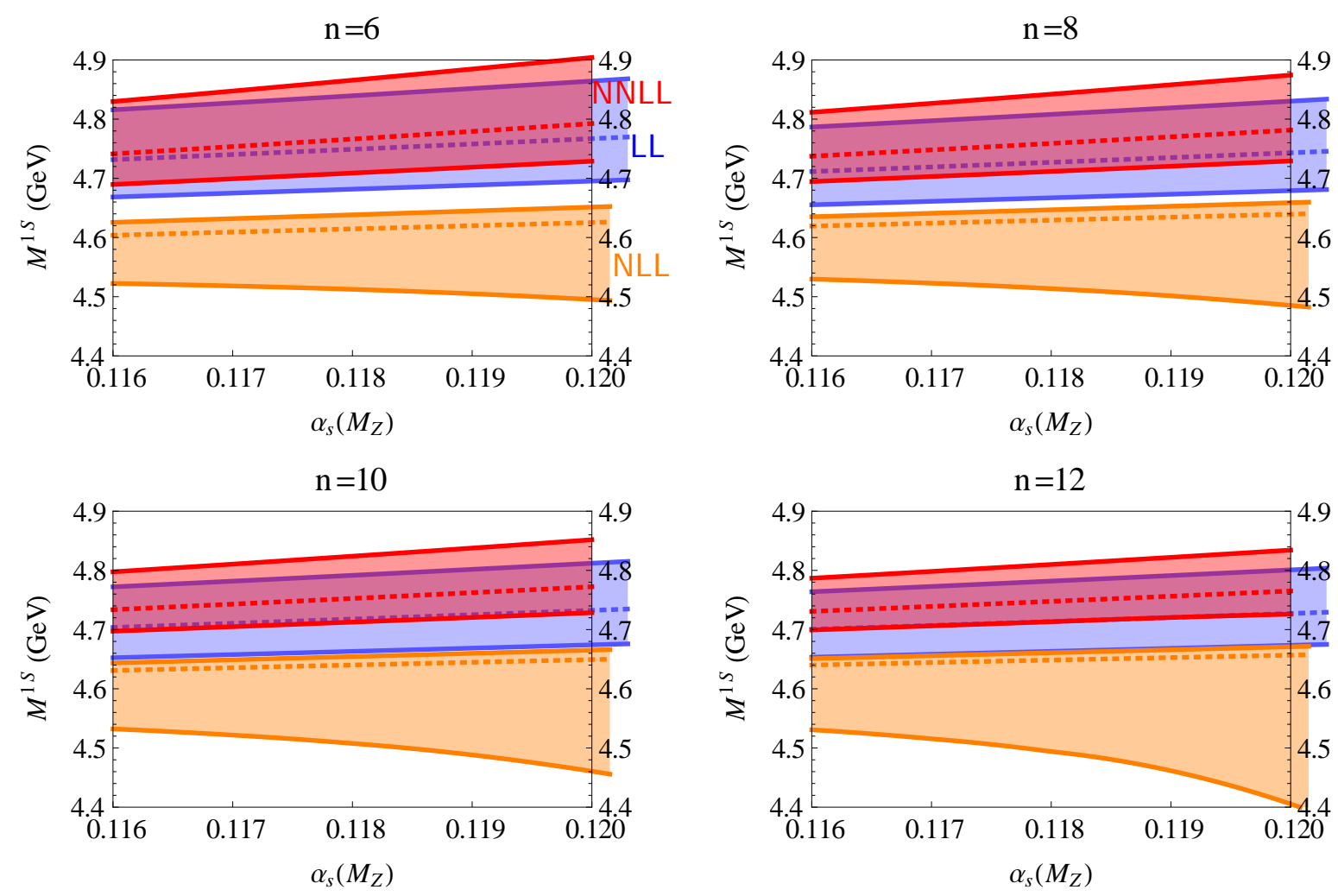

FIG. 5. $1 \mathrm{~S}$ bottom mass with errors determined from $P_{n}^{t h}\left(m_{b}\right)=P_{n}^{e x p}$ for $n=6,8,10,12$ as a function of $\alpha_{s}\left(M_{Z}\right)$ using the strictly expanded expression for $P_{n}^{t h}\left(m_{b}\right)$. As indicated in the panel for $n=6$ we plotted for each moment three (partly overlapping) bands with LL (blue), NLL (orange) and NNLL (red) precision, respectively. The error bands are generated by varying the parameters $f$ and $h$ of the theoretical moments $P_{n}^{t h}$ within the parameter space defined in Fig. 3 and adding the corresponding experimental error in quadrature.

In Fig. 6 we show the corresponding results for the 1S bottom mass using the factorized expression for the theoretical moments. Here the LL results are identical to the previous analysis based on the expanded moments. While the width of the NNLL order bands from scale variations are almost identical to the expanded moment analysis, we see that the NNLL order results for the mass values are somewhat smaller, but consistent within errors with the results for the expanded moments. The $n$-dependence of the mass values for $\alpha_{s}$ values around the world average is about three times larger than for the fully expanded moments. We note again, that the factorized moments have an inconsistent renormalization scaling behavior, and we therefore adopt the moments in the expanded form for the main numerical analysis. The consistency of the results from expanded and the factorized moments, on the other hand, indicates that the two results are in agreement within their perturbative uncertainties.

For quoting our final number for the 1S bottom quark mass we take the default value obtained from the 10-th NNLL order moment in expanded form as the central value and adopt half the size of the band from scale variation as the perturbative uncertainty. Using 

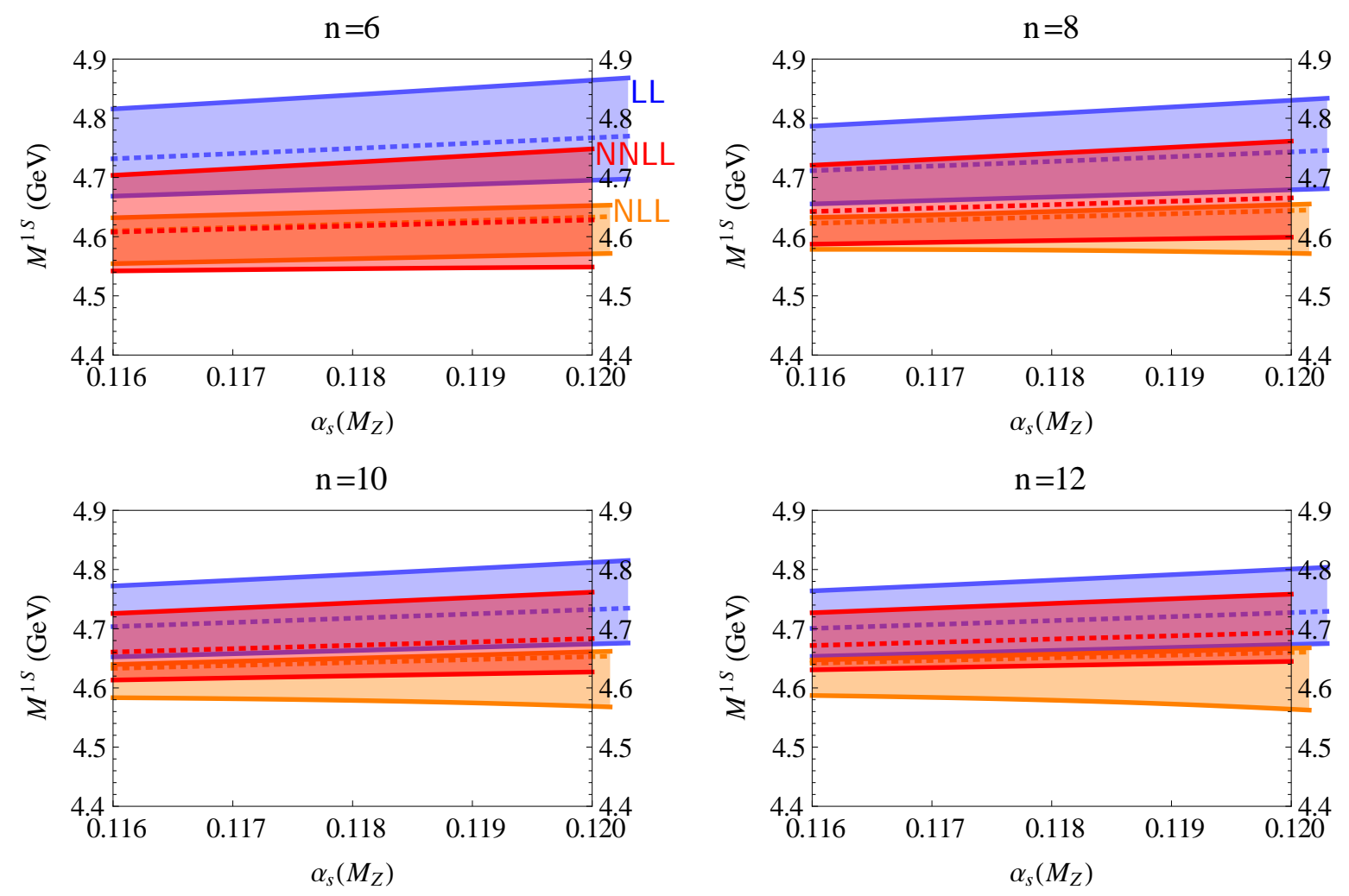

FIG. 6. Band plots analogous to Fig. 5 but with the factorized expression (as defined in the text) instead of the expanded expression used for the theoretical moments.

the current world average for the strong coupling $\alpha_{s}\left(M_{Z}\right)=0.1183 \pm 0.0010$ [39] we obtain

$$
M_{b}^{1 S}=4.755 \pm 0.057_{\text {pert }} \pm 0.009_{\alpha_{s}} \pm 0.003_{\exp } \mathrm{GeV}
$$

It is worth to emphasize that the $\alpha_{s}$ error increases by $9.3 \mathrm{MeV}$ for each unit of 0.001 the uncertainty in $\alpha_{s}\left(M_{Z}\right)$ is increased. It is also interesting to quote the result as a function of $\alpha_{s}\left(M_{Z}\right)$. Since the dependence of the bottom mass on the value of the strong coupling is linear to a very good approximation, we can quote the $\alpha_{s}$-dependence of the bottom $1 \mathrm{~S}$ mass by the following numerical fit formula:

$$
M_{b}^{1 S}=\left[4.755+\frac{\alpha_{s}\left(M_{Z}\right)-0.1183}{0.107}\right] \pm\left[0.057+\frac{\alpha_{s}\left(M_{Z}\right)-0.1183}{0.37}\right]_{\mathrm{pert}} \mathrm{GeV}
$$

where we have dropped the experimental error. The deviation of the linear approximation in Eq. (12) to the exact result is less than $0.6 \mathrm{MeV}$ for $0.113 \leq \alpha_{s}\left(M_{Z}\right) \leq 0.120$.

It is straightforward to convert our result for the $1 \mathrm{~S}$ mass to the $\overline{\mathrm{MS}}$ mass by combining the pole-1S mass relation in Eq. (6) and the pole- $\overline{\mathrm{MS}}$ mass relation. For this conversion one has to employ the $\epsilon$-expansion [17, 37, 38] to consistently cancel the renormalon contributions. The systematic cancellation of the renormalon contributions also requires that, within the conversion, the corrections in the pole-1S mass relation are evaluated at the same renormalization scale as those in the pole- $\overline{\mathrm{MS}}$ mass series. This can be achieved with the best convergence using the hard scale $M_{b}^{1 S}$ and requires to use the fixed order expansion 
for the pole-1S mass relation which is obtained from Eq. (6) for $\nu=1$ and $h=1$. Since the conversion formula has a significant $\alpha_{s}$-dependence due to a term $\propto \alpha_{s} m_{b}$ arising in the series relating the $\overline{\mathrm{MS}}$ and pole masses one needs to convert using the full $\alpha_{s}$-dependence of the 1S mass result as displayed in Eq. (12).

Using again the world average for the strong coupling as an input we then obtain

$$
\bar{m}_{b}\left(\bar{m}_{b}\right)=4.235 \pm 0.055_{\text {pert }} \pm 0.003_{\exp } \mathrm{GeV},
$$

where we have added an additional conversion error of $15 \mathrm{MeV}$ to the perturbative uncertainty coming from half the size of the three-loop correction $\bigsqcup^{4}$ in the perturbative series. The resulting value for the perturbative uncertainty is, however, still smaller than for the 1S mass due to the conversion series. The uncertainty coming from the value of $\alpha_{s}$ reduces to less than $0.5 \mathrm{MeV}$, as the sizable $\alpha_{s}$ dependence in the $1 \mathrm{~S}-\overline{\mathrm{MS}}$ conversion series mentioned above is anticorrelated to the monotonically increasing $\alpha_{s}$ dependence of the $1 \mathrm{~S}$ mass. Remarkably these two effects cancel almost entirely. We have therefore dropped the $\alpha_{s}$ induced error from Eq. (13). In analogy to Eq. (12) we can also give the result for the $\overline{\mathrm{MS}}$ mass showing the full $\alpha_{s}$ dependence as a linear fit function:

$$
\bar{m}_{b}\left(\bar{m}_{b}\right)=4.235 \pm\left[0.055+\frac{\alpha_{s}\left(M_{Z}\right)-0.1183}{0.41}\right]_{\text {pert }} \mathrm{GeV}
$$

where we did not include a linear $\alpha_{s}$ fit term for the central value as it contributes less than one $\mathrm{MeV}$ for $0.113 \leq \alpha_{s}\left(M_{Z}\right) \leq 0.120$. Again we have dropped the experimental error.

Our result for the bottom $\overline{\mathrm{MS}}$ mass $\bar{m}_{b}\left(\bar{m}_{b}\right)$ should be compared with the corresponding result obtained in the analysis of Ref. [22. Their analysis was also based on RGI large- $n$ moments having NNLL order input, but was using only the NLL order result for the anomalous dimension of the leading order current Wilson coefficient $c_{1}$. Thus their theoretical moments were missing the numerically important NNLL contribution to the evolution of $c_{1}$ shown in Fig. 1. Since $c_{1}$ enters as a square, their theoretical moments were by about $30 \%$ lower than ours. For a single fit for the 10-th moment one therefore expects, due to the $1 / m_{b}^{2 n}$ overall dependence on the mass as shown in Eq. (5), that their mass result is by about $1.3 \%$ lower than ours. This amounts to about $50 \mathrm{MeV}$ and is consistent with the result $\bar{m}_{b}\left(\bar{m}_{b}\right)=4.19 \pm 0.06 \mathrm{MeV}$ obtained in their analysis. The perturbative uncertainty estimated in their work was quoted between 45 and $65 \mathrm{MeV}$ and is numerically similar to ours. We note, however, that they determined the uncertainty from the difference in the bottom mass obtained from the NLL order and their incomplete NNLL order moments.

We also note that our result for the $\overline{\mathrm{MS}}$ mass is not quite compatible with the results given in Ref. [45] $\left(\bar{m}_{b}\left(\bar{m}_{b}\right)=4.163 \pm 16 \mathrm{MeV}\right)$ or even in Ref. [10] $\left(\bar{m}_{b}\left(\bar{m}_{b}\right)=4.171 \pm 9 \mathrm{MeV}\right)$ which were obtained from $\mathcal{O}\left(\alpha_{s}^{3}\right)$ fixed order analyses related to low- $n \Upsilon$ sum rules and taking their uncertainties exactly as quoted. Here we note again that we have treated the charm as a massless quark. In previous fixed-order analyses [18, 35] it was found that the non-zero charm mass causes shifts in the bottom quark mass obtained from large- $n$ sum rules between -20 and $-30 \mathrm{MeV}$. This sizable corrections is related to the fact that the charm mass is similar to the inverse Bohr radius $\alpha_{s} m_{b}$ and therefore not $1 / m_{b}$-suppressed. At this time the renormalization group improved non-relativistic effective theory approach has not yet been extended systematically to account for massive virtual quark thresholds. The required conceptual developments are theoretically interesting and might contribute reconciling the discrepancy. For comparing our result to bottom mass determinations from other physical quantities we refer to Ref. [13].

4 This corresponds to the $\mathcal{O}\left(\epsilon^{3}\right)$ terms in the epsilon expansion, see e.g. Eq. (99) of Ref. [18. 

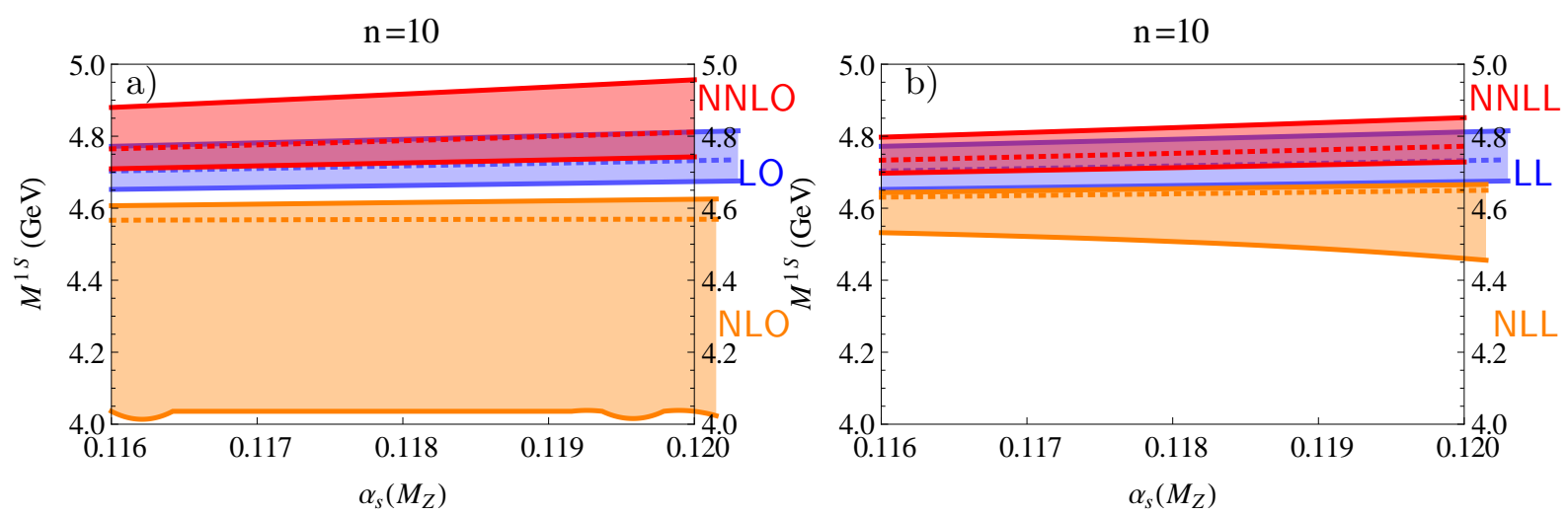

FIG. 7. Comparison of the masses obtained from the fixed order (a) and RGI calculation (b) of the 10-th moment, $P_{10}^{t h}\left(m_{b}\right)=P_{10}^{e x p}$. In panels a and $\mathrm{b}$ we show the mass values with LO, NLO, NNLO and LL, NLL, NNLL accuracy, respectively. The corresponding error bands were generated in the same way as in Fig. 5. Concerning panel a, we note that for some low $m_{b}$ values in the NLO band and the associated values for $h$ and $f$ the ultrasoft coupling $\alpha_{S}\left(\mu_{U}\right)$ reaches 0.65 causing numerical instabilities.

\section{FIXED-ORDER MOMENTS AND MULTIPLE MOMENT FITS}

Before RGI predictions based on the extended versions of NRQCD were possible a number of fixed-order analyses were carried out at the NNLO level [14-18]. As mentioned in Sec. I. the fixed-order moments showed a rather bad perturbative behavior, and the different analyses addressed this issue in different ways in order to achieve bottom mass determinations with small uncertainties. We can turn our RGI moments into fixed-order moments by setting the matching, soft and ultrasoft scales equal to the soft scale $\mu_{S}$, except for the current Wilson coefficients, where we keep the matching scale $\mu_{h}$. This leads to a logarithm $\ln \left(\mu_{S} / \mu_{h}\right)=\ln \left(f \nu^{*}\right)$ appearing in the current Wilson coefficient $c_{1}$ whose coefficient does not depend on the regularization scheme nor the conventions used for the potentials. Although the form of the resulting moments do not agree exactly with either one of the analyses in Refs. [14 18] due to differences in the regularization scheme, the expansion scheme or in the conventions for the potentials, the resulting fixed-order moments provide a good insight into the improvement obtained by the renormalization group logarithms summed into the RGI moments.

The $1 \mathrm{~S}$ bottom mass results obtained by carrying out a single moment fit for our fixedorder moment for $n=10$ in the expanded form and using the same variations of $h$ and $f$ we employed in the RGI analysis $5^{5}$ which now only cause correlated variations in the matching and the soft renormalization scale, is shown in Fig. 7. The result should be compared with the outcome using the RGI NNLL order moment $P_{10}^{t h}$ shown in the lower left panel of Fig. 5 . which we have for convenience displayed once more in the right panel of Fig. 7. Because at LL order the soft scale is the only renormalization scale that arises in the theoretical moment, the LL RGI and LO fixed-order results agree exactly. Substantial differences are,

5 The region in the $h-f$ plane we use in our analysis leads to the variations $0.75 m_{b} \leq \mu_{h} \leq 1.33 m_{b}$ and $0.32 m_{b} \leq \mu_{S} \leq 0.84 m_{b}$ for the matching and soft scales, respectively. For the matching scale the variation is somewhat smaller than what was employed in the fixed-order analysis of Refs. [14 18] but roughly agrees with those analyses for the soft scale. 
however, visible for the NLL vs. NLO results where the fixed order result shows even lower bottom mass values and a substantially larger scale variation. Comparing the NNLL and NNLO results we see that the fixed-order results cover larger bottom masses and exhibit scale variations that are twice as big as for the RGI results. We clearly see the improvement related to summing the logarithmic contributions within the RGI approach. Determining the final result for the $1 \mathrm{~S}$ bottom mass from the NNLO moment in the same way as we did it in our NNLL RGI analysis we would obtain for $\alpha_{s}\left(M_{Z}\right)=0.1183 \pm 0.001$ the result $M_{b}^{1 S}=4.791 \pm 0.097_{\text {pert }} \pm 0.012_{\alpha_{s}} \pm 0.003_{\text {exp }} \mathrm{GeV}$ (and thus $\bar{m}_{b}\left(\bar{m}_{b}\right)=4.269 \pm 0.090_{\text {pert }} \pm$ $0.002_{\alpha_{s}} \pm 0.003_{\exp } \mathrm{GeV}$ for the $\overline{\mathrm{MS}}$ mass), which is compatible with our results from Eqs. (11) and (13), but with a substantially larger perturbative uncertainty. Our fixed-order result is in agreement with Ref. [16] $\left(\bar{m}_{b}\left(\bar{m}_{b}\right)=4.26 \pm 0.09_{\text {pert }} \pm 0.01_{\alpha_{s}} \pm 0.02_{\text {exp }} \mathrm{GeV}\right)$ which also used a NNLO fixed-order single moment fit for $n=10$ and at the time of the analysis accounted for larger strong coupling and experimental errors.

Another instructive analysis is related to carrying out simultaneous fits to several moments using a $\chi^{2}$ analysis. It is straightforward to carry out such fits with our moments as well. In Fig. 8 we show the allowed bottom mass ranges obtained from $\chi^{2}$-fits based on the moments for $n=4,6,8,10$ where brown refers to the fixed-order NNLO analysis and, for comparison, red to the RGI NNLL order analysis. The upper panels show the outcome for the $n$-dependent assignment of the renormalization scales and the variation in the $h-f$ plane as described for the previous analyses, i.e. the choice of scales for the moments entering the $\chi^{2}$ function is $n$-dependent. Here the left panel is obtained using the full experimental covariance matrix (based on treating the experimental input data for masses, widths and $R$-ratio values as statistically independent), and the right panel is obtained treating the moments as statistically independent. The result for independent moments agrees almost exactly with the result of the $n=10$ single moment fit, which is easily understood since $P_{10}^{e x p}$ has the smallest error and thus the highest weight for uncorrelated moments.

Accounting for the correlation, on the other hand, leads to a different outcome, where the NNLL order bottom mass values are lower and the NNLO fixed order results lead to much smaller scale variations compared to the single moment or the uncorrelated fits. We have traced this behaviour back to the fact that the correlation coefficients are very close to unity and that the $\chi^{2}$ function fits the $n$-dependent shape of the moments rather than the actual values of the moments. Together with the correlated scale dependence of the theory moments this leads to the observed strong cancellation of the scale variation. This is also associated to rather large minimal $\chi^{2} /$ dof values with an average of around 50 . From this we conclude that the correlations are too large and the resulting scale variations are unreliable. Accounting in addition for the fact that the theory uncertainties are much bigger than the experimental ones, we conclude that single moment fits seem to be the only practical option to extract the bottom mass.

This conclusion is also reconfirmed by the analysis shown in the lower two panels. Here we have displayed the results for the bottom mass using the same $\chi^{2}$ fit method as for the respective upper two panels, but now using the renormalization scales adopted for $n=7$ (the average of the $n$ values used for the fit) for all moments. Treating the experimental moments as uncorrelated (right lower panel) the results for the bottom mass are very similar as for the moments with $n$-dependent scales (shown in the right upper panel) since again the 10-th moment has the highest weight in the fit. On the other hand, including the correlation we find that almost all scale variations cancel. For the NNLL moments the resulting scale variation is between 25 and $30 \mathrm{MeV}$, while for the NNLO moments it is below $5 \mathrm{MeV}$. This remarkable 
n-dependent $v$, full correlation
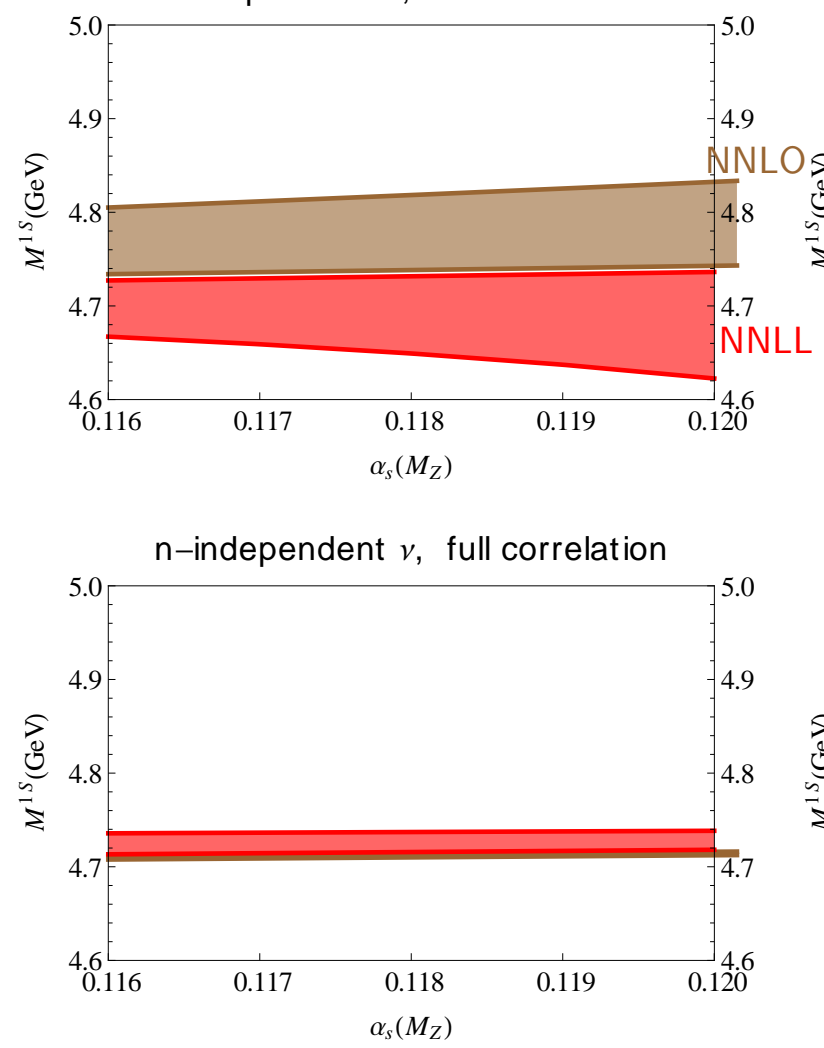
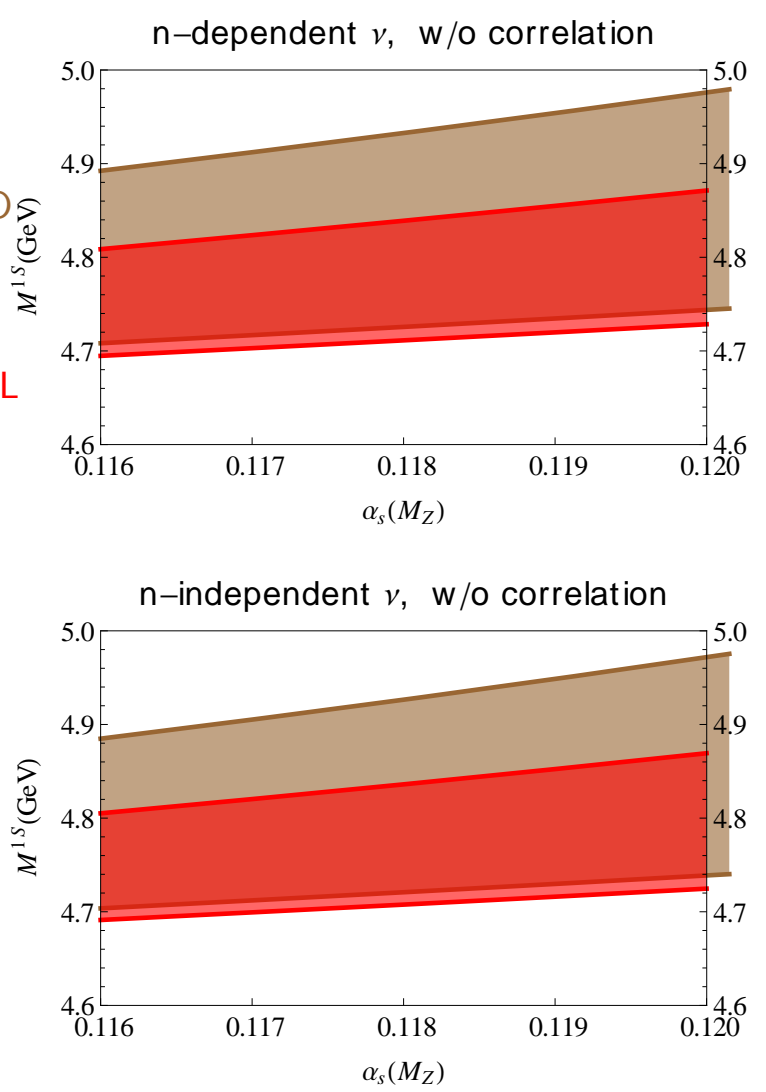

FIG. 8. Multiple moment fits for the $1 \mathrm{~S}$ mass with $n=\{4,6,8,10\}$. Each panel shows two bands associated with a fit using the NNLO fixed order moments (brown) and the NNLL RGI moments (red), respectively. The bands are generated by scanning the usual $h-f$ region (see Fig. 3). Fits scanning an $n$-dependent $\nu$ range with $\nu^{*}=(1 / \sqrt{n}+0.2)$ and an $n$-independent $\nu$ range with $\nu^{*}=(1 / \sqrt{7}+0.2)$ while allowing for full/no correlation of the experimental moments are shown as indicated by the labels of the individual panels.

cancellation can be traced back to the fact that the experimental correlation matrix puts the highest weight to a linear combination of moments that minimized the sensitivity to the uncertainties of the $\Upsilon$ electronic widths, which represent the biggest source of uncertainty for the large- $n$ experimental moments. Using the same renormalization scale values for all moments reduces very effectively the sensitivity to the electronic width of the perturbative $\Upsilon$ bound states entering the theoretical moments. Since the theoretical electronic widths, and the theoretical $R$-ratio in general, are the biggest source of scale variation in the theory moments, a common choice of renormalization scale in all moments, leads to the observed reduction of scale variation.

Given our observation that the experimental correlation of the moments is too strong to allow for reliable multiple moment fits, we have to conclude that the tiny scale variation shown in the lower left panel is an artifact of the scale choice and does not represent an estimate of the perturbative uncertainty. Overall the unreliable multiple moment fits are a consequence of the fact that the theory errors are significantly larger than the experimental ones. We note that fixed-order multiple moment fits with $n$-independent renormalization scales based on several different sets of moments were used in Refs. [14, 17, 18, The 
theoretical uncertainties quoted in these analyses originated mainly from using the different sets of moments and not from the scale variation.

\section{CONCLUSION}

In this work we have determined the $1 \mathrm{~S}$ bottom quark masses from large- $n \Upsilon$ sum rules with renormalization group improvement. Since for large values of $n$ the quark dynamics entering the moments is non-relativistic, the experimental moments are dominated by the well known data on the $\Upsilon$ resonances and the experimentally unmeasured continuum contributions are not relevant. The theoretical computation of the moments involves a simultaneous expansion in $\alpha_{s}$ and $1 / \sqrt{n}$ and is carried out within the framework of non-relativistic effective field theories. In general, within the non-relativistic framework where $n$ is large, one has to treat terms $\propto \alpha_{s} / \sqrt{n}$ and $\propto \alpha_{s} \ln (n)$ of order unity. For the renormalization group improved treatment both types of terms are summed to all orders following the generic scheme of Eq. (3). We have carried out this scheme using the vNRQCD ("velocity" NRQCD) formalism originally devised in Ref. [21] and extended in a number of subsequent publications as discussed in the main body of this paper. We have also determined the $\overline{\mathrm{MS}}$ bottom quark mass $\bar{m}_{b}\left(\bar{m}_{b}\right)$. We note that the conversion involves a sizable dependence on the value of the strong coupling $\alpha_{s}$. While we find that the bottom 1S mass slightly increases with $\alpha_{s}$, the $\overline{\mathrm{MS}}$ mass $\bar{m}_{b}\left(\bar{m}_{b}\right)$ turns out to be essentially $\alpha_{s}$-independent.

In this work we have for the first time carried out a renormalization group improved analysis using all available NNLL order results. At the NNLL order level currently all corrections are known except for the so-called soft mixing corrections to the anomalous dimension of the leading order heavy quark pair production current. We have given arguments showing that the known full set of NNLL order ultrasoft corrections numerically dominate over the soft contributions and that the uncertainty coming from the set of unknown soft corrections are negligible compared to the large ultrasoft contributions and their remaining scale variation at the NNLL order level. Our results can therefore be considered as the outcome of a full NNLL order analysis.

Prior to our work a number of large- $n$ analyses were carried out, which were based on the so-called fixed-order approach, where only the terms $\propto \alpha_{s} / \sqrt{n}$ were summed up systematically [14 17]. One important characteristic of these fixed-order analyses was that they exhibited rather bad convergence properties. We found that the summation of the logarithms contained in our moments improves the convergence considerably. Nevertheless the perturbative series for the renormalization group improved moments shows sizable corrections which made a careful examination of the perturbative uncertainty of our NNLL order prediction mandatory.

Our final results have been presented in Eqs. (11, 14) of Sec. IV.

\section{ACKNOWLEDGMENTS}

The work of P. R. is partially supported by MEC (Spain) under grants FPA200760323 and FPA2011-23778 and by the Spanish Consolider-Ingenio 2010 Programme CPAN (CSD2007-00042). We thank Vicent Mateu and Bahman Dehnadi for providing us with the code for the experimental moments and Vicent Mateu for discussion. 


\section{Appendix A: NNLL calculation of the moments}

The calculation of the contributions $\varrho_{n, 1}$ and $\varrho_{n, 2}$ to the $n$-th NNLL moment in Eq. (5) follows the lines of Ref. [14]. The results can be written as

$$
\begin{aligned}
\varrho_{n, 1}= & {\left[\varrho_{n, 1}\right]_{c}+\left[\varrho_{n, 1}\right]_{\mathrm{kin}}+\left(\mathcal{V}_{2}^{(s)}(\nu)+2 \mathcal{V}_{s}^{(s)}(\nu)\right)\left[\varrho_{n, 1}\right]_{\delta}+\mathcal{V}_{r}^{(s)}(\nu)\left[\varrho_{n, 1}\right]_{r} } \\
& -C_{A} C_{F} \alpha_{S}^{2}\left[\varrho_{n, 1}\right]_{\mathrm{CACF}}+\frac{C_{F}^{2}}{2} \alpha_{S}\left[\varrho_{n, 1}\right]_{\mathrm{CF} 2} \\
& +\alpha_{S}^{2} \mathcal{V}_{k 1}^{(s)}(\nu)\left[\varrho_{n, 1}\right]_{k 1}+\alpha_{S}^{2} \mathcal{V}_{k 2}^{(s)}(\nu)\left[\varrho_{n, 1}\right]_{k 2}
\end{aligned}
$$

and

$$
\varrho_{n, 2}=\frac{3}{4 n}\left[2+\frac{8}{3} \sqrt{\pi} \phi-4 \sqrt{\pi} \sum_{p=2}^{\infty} \phi^{p} \frac{2(p-3)}{3} \frac{\zeta_{p}}{\Gamma\left(\frac{p-1}{2}\right)}\right] .
$$

We abbreviate the strong coupling at the matching, soft and ultrasoft scales as $\alpha_{h}=\alpha_{s}\left(\mu_{h}\right)$, $\alpha_{S}=\alpha_{s}\left(\mu_{h} f \nu^{*}\right)$ and $\alpha_{U}=\alpha_{s}\left(\mu_{h} f^{2} \nu^{* 2}\right)$, respectively, and define

$$
\phi \equiv \frac{a \sqrt{n}}{2} \quad, \quad a \equiv-\frac{1}{4 \pi} \mathcal{V}_{c, \mathrm{eff}}^{(s)}(\nu)
$$

The expressions for the potential Wilson coefficients, $\mathcal{V}_{X}^{(s)}$, can be found in Ref. [32]. Except for the contribution of the Coulomb potential $\left[\varrho_{n, 1}\right]_{c}$, which contributes at LL and NLL order, all the other terms contained in Eqs. A1 A2) constitute NNLL order contributions. The LL Coulomb contribution reads

$$
\left[\varrho_{n, 1}\right]_{c}^{\mathrm{LL}}=1+2 \sqrt{\pi} \phi+4 \sqrt{\pi} \sum_{p=2}^{\infty} \phi^{p} \frac{\zeta_{p}}{\Gamma\left(\frac{p-1}{2}\right)} .
$$

and differs from the fixed order LO result given in Ref. [14] only through the definition of the variable $\phi$ which in the fixed-order treatment involves the replacement $a \rightarrow C_{F} \alpha_{s}\left(\mu_{S}\right)$. The difference constitutes a NNLL order correction. For the analytic expressions of the NLL and NNLL contributions to $\left[\varrho_{n, 1}\right]_{c}$ coming from the one- and two-loop corrections to the Coulomb potential we refer the reader to the expressions (57-63) in Ref. [14], where the identification $\mu_{\text {soft }}=\mu_{S}$ is understood.

The contribution to the Coulomb potential arising from the QED photon exchange is formally a NLL order correction, when we count the electromagnetic coupling $\alpha_{\mathrm{em}}$ as $\mathcal{O}\left(\alpha_{s}^{2}\right)$. The correction can be implemented in a straightforward way with the replacement $\mathcal{V}_{c, \text { eff }}^{(s)} \rightarrow \mathcal{V}_{c \text {,eff }}^{(s)}-4 \pi Q_{b}^{2} \alpha_{\text {em }}$ in Eq. A4). However, numerically one has to compare $C_{F} \alpha_{s}$ with $Q_{b} \alpha_{\mathrm{em}}$, and for the strong coupling at the soft scale one obtains that $\left(Q_{b} \alpha_{\mathrm{em}}\right) /\left(C_{F} \alpha_{S}\right)^{2}$ is $\mathcal{O}(1 \%)$ which makes QED effects entirely negligible for our NNLL order analysis. For an implementation of QED effects a fixed-order treatment is therefore well justified. Apart from the QED effect in the Coulomb potential one then only has to consider a NNLL order correction in the matching condition for the current coefficient $c_{1}$ given in Eq. (B11) through the replacement $C_{F} \alpha_{s} \rightarrow C_{F} \alpha_{s}+Q_{b} \alpha_{\text {em }}$ in the NLL order correction. We find that this affects the bottom quark mass by less then $1 \mathrm{MeV}$, which confirms the observation made in Ref. [22]. 
The remaining non-Coulombic contributions to $\varrho_{n, 1}$ differ from Ref. [14] because we use the differing conventions for the potentials from Refs. [27, 32] and dimensional regularization as the regularization scheme. The corresponding results are new and read

$$
\begin{gathered}
{\left[\varrho_{n, 1}\right]_{\mathrm{kin}}=\frac{1}{n}\left[-\frac{3}{8}-4 \sqrt{\pi} \sum_{p=2}^{\infty} \phi^{p} \frac{(p-1)(p-3)}{8} \frac{\zeta_{p}}{\Gamma\left(\frac{p-1}{2}\right)}\right]} \\
+\frac{2}{n} f_{n}(\phi)+\frac{a^{2}}{2}\left(\frac{3}{2}-\ln 2+\ln \left(\mu_{S} / m_{b}\right)\right)\left[\varrho_{n, 1}\right]_{c}^{\mathrm{LL}} \\
{\left[\varrho_{n, 1}\right]_{\delta}=-\frac{1}{4 \pi}\left[\frac{8}{a n} f_{n}(\phi)-2 a\left(-\frac{1}{2}+\ln 2-\ln \left(\mu_{S} / m_{b}\right)\right)\left[\varrho_{n, 1}\right]_{c}^{\mathrm{LL}}\right]} \\
{\left[\varrho_{n, 1}\right]_{r}=-\frac{1}{4 \pi}\left[\frac{8}{a n} f_{n}(\phi)-2 a\left(-\frac{3}{2}+\ln 2-\ln \left(\mu_{S} / m_{b}\right)\right)\left[\varrho_{n, 1}\right]_{c}^{\mathrm{LL}}\right]} \\
-\frac{1}{4 \pi a n}\left[2 \sqrt{\pi} \phi+4 \sqrt{\pi} \sum_{p=2}^{\infty} \phi^{p} \frac{\zeta_{p}(3-p) p}{2 \Gamma\left(\frac{p-1}{2}\right)}\right], \\
{\left[\varrho_{n, 1}\right]_{k 2}=-\frac{2}{a}\left[\frac{8}{a n} f_{n}(\phi)-2 a\left(-\frac{21}{16}+\ln 2-\ln \left(\mu_{S} / m_{b}\right)\right)\left[\varrho_{n, 1}\right]_{c}^{\mathrm{LL}}\right]} \\
{\left[\varrho_{n, 1}\right]_{\mathrm{CACF}}=-\frac{1}{2 a}\left[\frac{8}{a n} f_{n}(\phi)-2 a\left(-\frac{5}{4}+\ln 2-\ln \left(\mu_{S} / m_{b}\right)\right)\left[\varrho_{n, 1}\right]_{c}^{\mathrm{LL}}\right]} \\
{\left[\varrho_{n, 1}\right]_{\mathrm{CF} 2}=-\frac{1}{2 a}\left[\frac{8}{a n} f_{n}(\phi)-2 a\left(-1+\ln 2-\ln \left(\mu_{S} / m_{b}\right)\right)\left[\varrho_{n, 1}\right]_{c}^{\mathrm{LL}}\right]}
\end{gathered}
$$

with the function $f_{n}(\phi)$ defined as

$$
\begin{aligned}
f_{n}(\phi)= & \phi^{2}\left\{-1+\frac{\gamma_{\mathrm{E}}}{2}+\ln (2 \sqrt{n})+2 \sqrt{\pi} \phi\left(\frac{\gamma_{\mathrm{E}}}{2}+\ln \sqrt{n}\right)-2 \sqrt{\pi} \sum_{p=3}^{\infty} \phi^{p-1} \frac{\zeta_{p}}{\Gamma\left(\frac{p-2}{2}\right)}\right. \\
& \left.-4 \sqrt{\pi} \sum_{p=2}^{\infty} \phi^{p} \frac{\zeta_{p}}{\Gamma\left(\frac{p-1}{2}\right)}\left(\frac{1}{2} \Psi\left(\frac{p-1}{2}\right)-\ln \sqrt{n}\right)+2 \sqrt{\pi} \sum_{p, q=2}^{\infty} \phi^{p+q-1} \frac{\zeta_{p} \zeta_{q}}{\Gamma\left(\frac{p+q-2}{2}\right)}\right\} .
\end{aligned}
$$

We note that for the NNLL order correction terms in the moments it is sufficient to use $a \simeq a^{\mathrm{LL}}=C_{F} \alpha_{S}^{\mathrm{LL}}$ in Eqs. $\mathrm{A} 5$ - $\mathrm{A} 11$. 


\section{Appendix B: Current Wilson Coefficients}

The NLL anomalous dimension of the ${ }^{3} S_{1}$ current coefficient $c_{1}(\nu)$ is given by [32, 46, 47]

$$
\left(\nu \frac{\partial}{\partial \nu} \ln \left[c_{1}(\nu)\right]\right)^{\mathrm{NLL}}=-\frac{\mathcal{V}_{c}^{(s)}(\nu)}{16 \pi^{2}}\left[\frac{\mathcal{V}_{c}^{(s)}(\nu)}{4}+\mathcal{V}_{2}^{(s)}(\nu)+\mathcal{V}_{r}^{(s)}(\nu)+\mathbf{S}^{2} \mathcal{V}_{s}^{(s)}(\nu)\right]+\frac{1}{2} \mathcal{V}_{k, \text { eff }}^{(s)}(\nu)
$$

with $\mathbf{S}^{2}=2$ for the heavy quark pair produced in the spin triplet state. The solution at NLL order, $\xi^{\mathrm{NLL}}$, has been given in Eq. (57) of Ref. [32]. The form of NNLL ultrasoft mixing corrections in Eq. (B2) below implies the convention that the $z$ and $\omega=1 /(2-z)$ parameters in the expression for $\xi^{\mathrm{NLL}}$, as given in Ref. [32, are defined according to Eq. (B6) below.

The ultrasoft contribution to the NNLL mixing correction $\xi_{\mathrm{m}}^{\mathrm{NNLL}}$ in Eq. (8) was determined in Ref. 25] from Eq. (B1) through the subleading NLL ultrasoft RG evolution of the potentials $\mathcal{V}_{2}, \mathcal{V}_{r}$ and $\mathcal{V}_{k \text {,eff }}$ [24, 25] (see also Ref. [26]) and reads

$$
\begin{aligned}
\xi_{\mathrm{m}, \mathrm{usoft}}^{\mathrm{NNLL}}= & \frac{2 \pi \beta_{1}}{\beta_{0}^{3}} \tilde{A} \alpha_{h}^{2}\left[-\frac{7}{4}+\frac{\pi^{2}}{6}+z\left(1-\ln \frac{z}{2-z}\right)+z^{2}\left(\frac{3}{4}-\frac{1}{2} \ln z\right)\right. \\
& \left.-\ln ^{2}\left(\frac{z}{2}\right)+\ln ^{2}\left(\frac{z}{2-z}\right)-2 \operatorname{Li}_{2}\left(\frac{z}{2}\right)\right] \\
+ & \frac{8 \pi^{2}}{\beta_{0}^{2}} \tilde{B} \alpha_{h}^{2}\left[3-2 z-z^{2}-4 \ln (2-z)\right],
\end{aligned}
$$

with

$$
\begin{aligned}
\beta_{0} & =\frac{11}{3} C_{A}-\frac{4}{3} T n_{f}, \quad \beta_{1}=\frac{34}{3} C_{A}^{2}-4 C_{F} T n_{f}-\frac{20}{3} C_{A} T n_{f}, \\
\tilde{A} & =-C_{F}\left(C_{A}+C_{F}\right)\left(C_{A}+2 C_{F}\right) \frac{1}{3 \pi}, \\
\tilde{B} & =-C_{F}\left(C_{A}+C_{F}\right)\left(C_{A}+2 C_{F}\right) \frac{C_{A}\left(47+6 \pi^{2}\right)-10 n_{f} T}{108 \pi^{2}}, \\
z & \equiv \frac{\alpha_{S}^{\mathrm{LL}}}{\alpha_{h}}=\left(1+\frac{\alpha_{h} \beta_{0}}{2 \pi} \ln \nu\right)^{-1} .
\end{aligned}
$$

The complete result for the soft NNLL mixing correction, $\xi_{\mathrm{m}, \mathrm{soft}}^{\mathrm{NLL}}$ is currently unknown. However, its linear logarithmic contribution $\propto \alpha_{h}^{3} \ln \nu$ was determined in Ref. [27] from the known subleading matching conditions for the potentials [48, 49] that appear in Eq. (B1) and reads

$$
\xi_{\mathrm{m}, \mathrm{soft} 1}^{\mathrm{NNL}}=\frac{\alpha_{h}^{3}}{48 \pi} C_{F}^{2}\left[C_{A}\left(16 \mathbf{S}^{2}-3\right)+4 C_{F}\left(5-2 \mathbf{S}^{2}\right)-\frac{16}{5} T_{F}\right] \ln \nu .
$$

The NNLL non-mixing contributions $\xi_{\mathrm{nm}}^{\mathrm{NNLL}}$ were determined in Ref. 27] for the assignment $\mu_{U}=\mu_{S}^{2} / m_{b}$, i.e. for $h=1$. For the more general case $\mu_{U}=\mu_{S}^{2} /\left(h m_{b}\right)$ we use in our work (see the discussion in Sec. II), the NNLL order non-mixing anomalous dimension receives an additional additive term $\propto \ln h$ which reads

$$
\delta \xi_{\mathrm{nm}}^{\mathrm{NNLL}}(h)=\frac{4 C_{F}\left(C_{A}^{2}+3 C_{A} C_{F}+2 C_{F}^{2}\right)}{3 \beta_{0}} \alpha_{h}^{2} \ln (h)\left(2 \ln \left[\frac{1}{2-z}\right]-z+1\right) .
$$


For a comparison carried out in our numerical analysis we also give the linear soft non-mixing logarithm contained in $\xi_{\mathrm{nm}}^{\mathrm{NNLL}}$ for $h=1$, which is determined by expanding the full result for $\xi_{\mathrm{nm}, \mathrm{soft}}^{\mathrm{NNLL}} \alpha_{h}$ :

$$
\xi_{\mathrm{nm}, \mathrm{soft} 1}^{\mathrm{NNLL}}=\frac{\alpha_{h}^{3}}{288 \pi} C_{F}\left[303 C_{A}^{2}+\left[8 C_{F}\left(\mathbf{S}^{2}-12\right)-111 C_{A}\right] \beta_{0}+2 C_{A} C_{F}\left(141-40 \mathbf{S}^{2}\right)+360 C_{F}^{2}\right] \ln \nu .
$$

Taking $\xi_{\mathrm{m}, \mathrm{soft} 1}^{\mathrm{N} L \mathrm{~L}}$ as an approximation for the complete soft NNLL mixing corrections, we can thus write the NNLL contribution to $\ln \left[c_{1}(\nu) / c_{1}(1)\right]$ for arbitrary $h$ as

$$
\xi^{\mathrm{NNLL}}=\left[\xi_{\mathrm{nm}}^{\mathrm{NNLL}}(h=1)+\delta \xi_{\mathrm{nm}}^{\mathrm{NNLL}}(h)\right]+\left[\xi_{\mathrm{m}, \mathrm{usoft}}^{\mathrm{NNLL}}+\xi_{\mathrm{m}, \mathrm{soft} 1}^{\mathrm{NNLL}}\right] .
$$

The matching condition for the current Wilson coefficient $c_{1}(1)$ is given in Ref. [27] for $h=1$. For arbitrary $h$ we have to add a $\ln (h)$ term which arises from the term $\ln \left(\mu_{h} / m_{b}\right)$ and find

$$
\begin{aligned}
c_{1}(1)= & 1-\frac{2 C_{F}}{\pi} \alpha_{h}+\alpha_{h}^{2}\left[C_{F}^{2}\left(\frac{\ln 2}{3}-\frac{31}{24}-\frac{2}{\pi^{2}}\right)+C_{A} C_{F}\left(\frac{\ln 2}{2}-\frac{5}{8}\right)+\frac{\kappa}{2}\right] \\
& -\alpha_{h}^{2} C_{F} \ln (h)\left[\frac{\beta_{0}}{\pi^{2}}+\frac{C_{A}}{2}+\frac{C_{F}}{3}\right],
\end{aligned}
$$

where the constant $\kappa$ was defined in Eq. (21) of Ref. [50].

The coefficient of the $v^{2}$ suppressed ${ }^{3} S_{1}$ current $c_{2}(\nu)$ is needed at the LL level and reads 31 ]

$$
c_{2}(\nu)=-\frac{8 C_{F}}{3 \beta_{0}} \ln \left(\frac{\alpha_{U}}{\alpha_{h}}\right)-\frac{1}{6}
$$

\section{Appendix C: Experimental moments}

The experimental moments we use in this work together with their statistical and systematic errors, respectively, read

$$
\begin{aligned}
& P_{4}^{e x p}=(2.17025 \pm 0.0128186 \pm 0.0464299) \times 10^{-9}, \\
& P_{5}^{\text {exp }}=(2.11692 \pm 0.0135132 \pm 0.0363657) \times 10^{-11}, \\
& P_{6}^{\text {exp }}=(2.13921 \pm 0.0144643 \pm 0.0317105) \times 10^{-13}, \\
& P_{7}^{\text {exp }}=(2.21297 \pm 0.0156671 \pm 0.0297788) \times 10^{-15}, \\
& P_{8}^{\text {exp }}=(2.32718 \pm 0.017121 \pm 0.029311) \times 10^{-17}, \\
& P_{9}^{\text {exp }}=(2.47683 \pm 0.0188304 \pm 0.0297155) \times 10^{-19}, \\
& P_{10}^{\text {exp }}=(2.66003 \pm 0.0208057 \pm 0.0307192) \times 10^{-21}, \\
& P_{11}^{\text {exp }}=(2.87678 \pm 0.0230625 \pm 0.032201) \times 10^{-23}, \\
& P_{12}^{\text {exp }}=(3.12831 \pm 0.0256223 \pm 0.0341133) \times 10^{-25}, \\
& P_{13}^{\text {exp }}=(3.41679 \pm 0.0285116 \pm 0.0364466) \times 10^{-27}, \\
& P_{14}^{\text {exp }}=(3.74516 \pm 0.0317625 \pm 0.0392118) \times 10^{-29} .
\end{aligned}
$$


For our correlated fits we have determined the covariance matrix according to the rules provided in statistics review of Ref. [13] treating the 10\% uncertainty in the contribution of the continuum region for $\sqrt{s}>11.21 \mathrm{GeV}$ as a single independent error source. Explicit formulae for the covariance matrix can also be found in Ref. [14].

[1] M. Voloshin, "On Dynamics of Heavy Quarks in Nonperturbative QCD Vacuum", Nucl.Phys. B154 (1979) 365.

[2] L. Reinders, H. Rubinstein, and S. Yazaki, "QCD Sum Rules for Heavy Quark Systems", Nucl.Phys. B186 (1981) 109.

[3] M. Beneke, "A Quark mass definition adequate for threshold problems", Phys.Lett. B434 (1998) 115-125, arXiv: hep-ph/9804241.

[4] A. Pineda, "Determination of the bottom quark mass from the Upsilon(1S) system", JHEP 0106 (2001) 022, arXiv:hep-ph/0105008.

[5] A. Hoang and T. Teubner, "Top quark pair production close to threshold: Top mass, width and momentum distribution", Phys.Rev. D60 (1999) 114027, arXiv:hep-ph/9904468.

[6] V. Novikov, L. Okun, M. A. Shifman, A. Vainshtein, M. Voloshin, et al., "Charmonium and Gluons: Basic Experimental Facts and Theoretical Introduction", Phys.Rept. 41 (1978) $1-133$.

[7] M. Voloshin, "Precision determination of alpha-s and $\mathrm{m}(\mathrm{b})$ from QCD sum rules for $\mathrm{b}$ anti-b", Int.J.Mod.Phys. A10 (1995) 2865-2880, arXiv:hep-ph/9502224.

[8] G. Corcella and A. Hoang, "Uncertainties in the MS-bar bottom quark mass from relativistic sum rules", Phys.Lett. B554 (2003) 133-140, arXiv:hep-ph/0212297.

[9] K. Chetyrkin, J. Kuhn, A. Maier, P. Maierhofer, P. Marquard, et al., "Precise Charm- and Bottom-Quark Masses: Theoretical and Experimental Uncertainties", Theor.Math.Phys. 170 (2012) 217-228, arXiv:1010.6157.

[10] S. Bodenstein, J. Bordes, C. Dominguez, J. Penarrocha, and K. Schilcher, "Bottom-quark mass from finite energy QCD sum rules", Phys.Rev. D85 (2012) 034003, arXiv:1111.5742.

[11] G. T. Bodwin, E. Braaten, and G. P. Lepage, "Rigorous QCD predictions for decays of P wave quarkonia", Phys.Rev. D46 (1992) 1914-1918, arXiv:hep-lat/9205006.

[12] A. Hoang, M. Beneke, K. Melnikov, T. Nagano, A. Ota, et al., "Top - anti-top pair production close to threshold: Synopsis of recent NNLO results", Eur.Phys.J.direct C2 (2000) 1, arXiv:hep-ph/0001286

[13] Particle Data Group Collaboration, J. Beringer et al., "Review of Particle Physics (Particle Data Group)", Phys.Rev. D86 (2012) 010001.

[14] A. Hoang, "Bottom quark mass from Upsilon mesons", Phys.Rev. D59 (1999) 014039, arXiv:hep-ph/9803454.

[15] K. Melnikov and A. Yelkhovsky, "The b quark low scale running mass from Upsilon sum rules", Phys.Rev. D59 (1999) 114009, arXiv:hep-ph/9805270.

[16] M. Beneke and A. Signer, "The Bottom MS-bar quark mass from sum rules at next-to-next-to-leading order", Phys.Lett. B471 (1999) 233-243, arXiv: hep-ph/9906475.

[17] A. Hoang, "1S and MS-bar bottom quark masses from Upsilon sum rules", Phys.Rev. D61 (2000) 034005, arXiv:hep-ph/9905550.

[18] A. Hoang, "Bottom quark mass from Upsilon mesons: Charm mass effects", arXiv:hep-ph/0008102. 
[19] M. Battaglia, A. Buras, P. Gambino, A. Stocchi, D. Abbaneo, et al., "The CKM matrix and the unitarity triangle. Workshop, CERN, Geneva, Switzerland, 13-16 Feb 2002:

Proceedings", arXiv:hep-ph/0304132.

[20] A. Pineda and J. Soto, "Effective field theory for ultrasoft momenta in NRQCD and NRQED", Nucl.Phys.Proc.Suppl. 64 (1998) 428-432, arXiv:hep-ph/9707481.

[21] M. E. Luke, A. V. Manohar, and I. Z. Rothstein, "Renormalization group scaling in nonrelativistic QCD", Phys.Rev. D61 (2000) 074025, arXiv:hep-ph/9910209.

[22] A. Pineda and A. Signer, "Renormalization group improved sum rule analysis for the bottom quark mass", Phys.Rev. D73 (2006) 111501, arXiv: hep-ph/0601185.

[23] A. Pineda and A. Signer, "Heavy Quark Pair Production near Threshold with Potential Non-Relativistic QCD", Nucl.Phys. B762 (2007) 67-94, arXiv:hep-ph/0607239.

[24] A. H. Hoang and M. Stahlhofen, "Two-loop ultrasoft running of the O(v**2) QCD quark potentials", Phys.Rev. D75 (2007) 054025, arXiv:hep-ph/0611292.

[25] A. H. Hoang and M. Stahlhofen, "Ultrasoft NLL Running of the Nonrelativistic O(v) QCD Quark Potential", JHEP 1106 (2011) 088, arXiv:1102.0269.

[26] A. Pineda, "Next-to-leading ultrasoft running of the heavy quarkonium potentials and spectrum: Spin-independent case", Phys.Rev. D84 (2011) 014012, arXiv:1101.3269.

[27] A. H. Hoang, "Three loop anomalous dimension of the heavy quark pair production current in nonrelativistic QCD", Phys.Rev. D69 (2004) 034009, arXiv:hep-ph/0307376.

[28] A. A. Penin, A. Pineda, V. A. Smirnov, and M. Steinhauser, "Spin dependence of heavy quarkonium production and annihilation rates: Complete next-to-next-to-leading logarithmic result", Nucl. Phys. B699 (2004) 183-206, hep-ph/0406175.

[29] A. Hoang and M. Stahlhofen, "NNLL top-antitop production at threshold", arXiv:1111.4486.

[30] A. Hoang, A. Manohar, I. W. Stewart, and T. Teubner, "A Renormalization group improved calculation of top quark production near threshold", Phys.Rev.Lett. 86 (2001) 1951-1954, arXiv:hep-ph/0011254.

[31] A. Hoang, A. Manohar, I. W. Stewart, and T. Teubner, "The Threshold t anti-t cross-section at NNLL order", Phys.Rev. D65 (2002) 014014, arXiv:hep-ph/0107144.

[32] A. H. Hoang and I. W. Stewart, "Ultrasoft renormalization in nonrelativistic QCD", Phys.Rev. D67 (2003) 114020, arXiv:hep-ph/0209340.

[33] A. Pineda and J. Soto, "The Renormalization group improvement of the QCD static potentials", Phys.Lett. B495 (2000) 323-328, arXiv:hep-ph/0007197.

[34] A. H. Hoang, A. V. Manohar, and I. W. Stewart, "The Running Coulomb potential and Lamb shift in QCD", Phys.Rev. D64 (2001) 014033, arXiv:hep-ph/0102257.

[35] A. Hoang and A. Manohar, "Charm effects in the MS-bar bottom quark mass from Upsilon mesons", Phys.Lett. B483 (2000) 94-98, arXiv:hep-ph/9911461.

[36] A. H. Hoang, A. Jain, I. Scimemi, and I. W. Stewart, "Infrared Renormalization Group Flow for Heavy Quark Masses", Phys.Rev.Lett. 101 (2008) 151602, arXiv:0803.4214.

[37] A. H. Hoang, Z. Ligeti, and A. V. Manohar, "B decay and the Upsilon mass", Phys.Rev.Lett. 82 (1999) 277-280, arXiv:hep-ph/9809423.

[38] A. H. Hoang, Z. Ligeti, and A. V. Manohar, "B decays in the upsilon expansion", Phys.Rev. D59 (1999) 074017, arXiv:hep-ph/9811239

[39] S. Bethke, A. H. Hoang, S. Kluth, J. Schieck, I. W. Stewart, et al., "Workshop on Precision Measurements of $\alpha_{s} "$, arXiv:1110.0016.

[40] A. Pineda, "Next-to-leading nonperturbative calculation in heavy quarkonium", Nucl.Phys. 
B494 (1997) 213-236, arXiv:hep-ph/9611388.

[41] BABAR Collaboration Collaboration, B. Aubert et al., "Measurement of the $e^{+} e^{-} \rightarrow b \bar{b}$ cross section between $\sqrt{s}=10.54-\mathrm{GeV}$ and 11.20-GeV", Phys.Rev.Lett. 102 (2009) 012001, arXiv:0809.4120.

[42] K. Chetyrkin, A. Hoang, J. H. Kuhn, M. Steinhauser, and T. Teubner, "Massive quark production in electron positron annihilation to order alpha (s)**2", Eur.Phys.J. C2 (1998) 137-150, arXiv:hep-ph/9711327.

[43] B. Dehnadi, A. H. Hoang, V. Mateu, and S. M. Zebarjad, "Charm Mass Determination from QCD Charmonium Sum Rules at Order $a l p h a_{s}^{3} "$, arXiv:1102.2264.

[44] J. H. Kuhn, M. Steinhauser, and C. Sturm, "Heavy Quark Masses from Sum Rules in Four-Loop Approximation", Nucl.Phys. B778 (2007) 192-215, arXiv:hep-ph/0702103.

[45] K. Chetyrkin, J. Kuhn, A. Maier, P. Maierhofer, P. Marquard, et al., "Charm and Bottom Quark Masses: An Update", Phys.Rev. D80 (2009) 074010, arXiv:0907.2110.

[46] A. V. Manohar and I. W. Stewart, "Running of the heavy quark production current and 1 / v potential in QCD", Phys.Rev. D63 (2001) 054004, arXiv:hep-ph/0003107.

[47] A. Pineda, "Next-to-leading log renormalization group running in heavy-quarkonium creation and annihilation", Phys.Rev. D66 (2002) 054022, arXiv:hep-ph/0110216.

[48] A. V. Manohar and I. W. Stewart, "The QCD heavy quark potential to order v**2: One loop matching conditions", Phys.Rev. D62 (2000) 074015, arXiv:hep-ph/0003032.

[49] B. A. Kniehl, A. A. Penin, M. Steinhauser, and V. A. Smirnov, "NonAbelian alpha**3(s) / (m(q)r**2) heavy quark anti-quark potential", Phys.Rev. D65 (2002) 091503, arXiv:hep-ph/0106135.

[50] A. Hoang and T. Teubner, "Top quark pair production at threshold: Complete next-to-next-to-leading order relativistic corrections", Phys.Rev. D58 (1998) 114023, arXiv:hep-ph/9801397 